\title{
Investigation of the Turbulent Flame Structure and Topology at Different Karlovitz Numbers Using the Tangential Stretching Rate Index
}

\author{
Dimitris M. Manias ${ }^{\mathrm{a}, \mathrm{b}}$, Efstathios-Al. Tingas ${ }^{\mathrm{a}, *}$, Francisco E. Hernández Pérez ${ }^{\mathrm{a}}$, Riccardo \\ Malpica Galassic ${ }^{c}$, Pietro Paolo Ciottolic ${ }^{\mathrm{c}}$, Mauro Valoranic ${ }^{\mathrm{c}}$, Hong G. Im ${ }^{\mathrm{a}}$ \\ ${ }^{a}$ King Abdullah University of Science and Technology (KAUST), Clean Combustion Research Center \\ (CCRC), Thuwal, 23955-6900, Saudi Arabia \\ ${ }^{b}$ Department of Mechanics, School of Applied Mathematical and Physical Sciences, National Technical \\ University, Athens 15773, Greece \\ ${ }^{c}$ Mechanical and Aerospace Engineering Department, Sapienza University, Rome 00184, Italy
}

\begin{abstract}
Turbulent premixed flames at high Karlovitz numbers exhibit highly complex structures in different reactive scalar fields to the extent that the definition of the flame front in an unambiguous manner is not straightforward. This poses a significant challenge in characterizing the observable turbulent flame behaviour such as the flame surface density, turbulent burning velocity, and so on. Turbulent premixed flames are reactive flows involving physical and chemical processes interacting over a wide range of time scales. By recognizing the multi-scale nature of reactive flows, we analyze the topology and structure of two direct numerical simulation cases of turbulent $\mathrm{H}_{2}$ /air premixed flames, in the thin reaction zone and distributed combustion regimes, using tools derived from the computational singular perturbation (CSP) method and augmented by the tangential stretching rate (TSR) analysis. CSP allows to identify the local time scale decomposition of the multi-scale problem in its slow and fast components, while TSR allows to identify the most energetic time scale during both the explosive and dissipative regime of the reactive flow dynamics together with the identification of the flame front in an unambiguous manner. Before facing the complexity of the turbulent flow regime, we carry out a preliminary analysis of a one-dimensional laminar premixed flame in view of highlighting similarities and differences between laminar and turbulent cases. Subsequently, it is shown that the TSR metric provides a reliable way to identify the turbulent flame topologies.
\end{abstract}

Keywords: CSP, TSR, extreme combustion, turbulent flames, high Karlovitz number, flame topology

\section{Introduction}

With the advances in supercomputing power, today's direct numerical simulations (DNS) commonly reproduce turbulent premixed flames incorporating detailed chemistry [1] at high Karlovitz numbers (Ka). Consistent with the theory of turbulent combustion regimes [2], recent simulations reported that the flames at such extreme conditions exhibit highly complex

*Corresponding author: stathis.tingas@kaust.edu.sa

Preprint submitted to Combustion and Flame 
structures in different reactive scalar fields, such that even a flame front cannot be identified in an unambiguous manner since it depends strongly on the specific choice of the sampling variable and its iso-surface values. This poses a significant challenge in characterizing the integrated turbulent flame observables, such as the flame surface density, turbulent burning velocity, and the like. The process of identification and quantification of turbulent flame quantities of interest in datasets generated by reacting flow models of constantly increasing size poses a further technical hurdle.

Reactive flows involve transport and chemical processes proceeding at vastly different paces occurring over time scales which differ by several orders of magnitude. Kinetics is usually the cause of the emergence of very fast time scales, while transport processes usually proceed at slower time scales. It is also quite common to observe a strong coupling between kinetics and transport at both fast and slow scales.

The dynamics of a multi-scale reactive flow problem can be significantly simplified when a spectral gap between slow and fast processes develops. Let us consider that in the $N$ dimensional chemical composition space (CCS), a spectral gap exists between the $M$-th and $(M+1)$-th time scale, and that the scales from 1 to $M$ are fast and the scales from $M+1$ to $N$ are slow, with a large gap in between. After a time period of the order of the fastest of the slow scales, i.e., the $(M+1)$-th scale, the $M$ fast processes attain a quasi-stationary (near equilibrium) state as a result of their mutual competition. The locus of state points where this near-equilibrium condition is attained forms a $(N-M)$-dimensional manifold in the $N$-dimensional chemical composition space, and this manifold constitutes the landscape over which the processes proceeding at the slow scales drive the system towards a possible global equilibrium or other asymptotic attractive loci such as limit cycles or chaotic attractors.

Under these circumstances, the effective dimension $(N-M)$ of the multi-scale reactive flow problem can become much smaller than the original dimension $N$ when the number of fast scales $M$ is large, or in other words, the effective number of degrees of freedom of the multi-scale problem becomes significantly smaller than $N$ because the slow evolution of the system is constrained within the $(N-M)$-dimensional slow invariant manifold (SIM). In chemical kinetics as an example, fast reactions in near equilibrium are those responsible for the emergence of the SIM, while chain branching and termination reactions are responsible for the slow motion constrained within the SIM.

This conceptual framework suggests that the dynamics of a reactive system will be fully characterized after having identified the role of each reaction in the emergence of the SIM, in the slow kinetic evolution, or its possible irrelevance in the given condition. Translating this concept into quantitative estimates involves specific mathematical and computational techniques. In this regard, the computational singular perturbation (CSP) [3, 4] method is grounded on asymptotic concepts, allowing a systematic analysis of a multi-scale system independently of its dimension, complexity, and degree of nonlinearity.

The CSP algorithm assumes that it is possible to identify a local decomposition of the tangent space in a $(N-M)$-dimensional slow and a $M$-dimensional fast subspaces. A new frame of reference is introduced in the $N$-dimensional CCS such that $N-M$ basis vectors span the slow subspace and $M$ basis vectors span the fast subspace. A leading order approximation of the basis vectors that ensure the best decoupling between slow and fast scales is obtained by taking the set of right eigenvectors of the Jacobian matrix of the vector field of the problem of interest as basis vectors. Higher order accuracy (accounting for nonlinearities) can be achieved by resorting to a procedure referred to as CSP refinements, which is equivalent to a block-power method $[4,5]$. 
The CSP decomposition allows to project the vector field onto the slow and fast subspaces. The fast projection of the vector field attains a vanishingly small magnitude over a time period of the order of the fastest of the slow scales. Effectively, the loci of state points where the fast projection of the vector field is nearly zero (thus a differential equation degenerates to an algebraic equation) identifies the SIM. The slow projection of the vector field defines a lower dimensional problem freed from all the fast scales, which evolves within the SIM.

CSP has been successfully used in combustion problems such as autoignition [6-9] and flames [10-13], chemical mechanism simplification [13-18], and as mathematical framework to develop stiff-savvy solvers [19-21] of the explicit type.

More recently, the identification of the slow scales that contribute the most to the system dynamics was achieved by the concept of tangential stretching rate (TSR) [22], which combines the amplitude with the time scale of slow (explosive and contractive) eigen-modes in a single metric, thus providing a unified and convenient characterization of the slow dynamics in terms of the most energy-containing scale, where the energy is the square of the mode amplitude. The TSR is essentially a time scale obtained as a weighted average of all slow time scales [23], with the weight depending on the amplitude associated with that scale, and on the relative orientation of the direction of the basis vector associated with that scale and the direction of the vector field. The TSR concept has been extended to cope with spatially inhomogeneous problems [24], so as to investigate flames for which both chemistry and transport play an important role.

Of all the CSP modes that characterize the dynamics of a system of reactive flows, the explosive ones are usually the most important ones, since they relate to components of the system that tend to drive it away from equilibrium. In the absence of transport, explosive modes are essential for ignition. However, in flames diffusion also plays a significant role to the ignition of the system, despite its dissipative nature. Note that an explosive mode can act as the dominant/characteristic mode driving the evolution of the system only when both of the following two conditions are satisfied:

i) its time scale is among the fastest of the slow ones, and

ii) its mode amplitude has the largest magnitude among all modes.

When either of the two conditions is not satisfied, other modes must be taken into account, which can be of explosive or dissipative nature. Therefore, the consideration of an explosive mode as the characteristic mode for the evolution of the system is not straightforward and must be subject to investigation.

Last but not least, explosive modes exist only in finite regions of the domain, and therefore, no information is provided about the dynamics of the system when or where they do not exist.

Our first attempt to identify TSR signatures in different combustion regimes were recently reported [24, 25], demonstrating the value of the TSR analysis in providing reactive-diffusive dynamics within turbulent flames. However, the study was limited mostly to cases in the flamelet regime at moderate Karlovitz numbers, where the basic one-dimensional flame normal structure is preserved against the turbulent forcing. Therefore, the present study aims to undertake the combined CSP and TSR analyses in identifying the flame topologies and the dominant processes on regard of turbulent premixed flames, over a wider range of turbulent conditions. The DNS datasets considered in this work have been generated and discussed in 
previous studies $\left[26,27\right.$ for statistically stationary $\mathrm{H}_{2}$ /air premixed flames, representative of the wrinkled flamelet and distributed combustion regimes.

With this background, the main goals of this work are:

(i) to perform a comparison of the flame front topology and structure in different turbulent regimes,

(iii) to analyze the flame dynamics by means of the TSR concept and the TSR-based indices.

Both goals are accomplished by comparing the spatial distribution of TSR as an effective means to identify the flame front region. It will be shown that the TSR metric is a reliable way to identify the turbulent flame topologies and it can capture the broadening of the reaction zone regime, which is a distinct feature of the broken reaction zone combustion regime.

\section{Theoretical Framework}

The dynamics of the scalar fields $\phi$ in the reactive Navier Stokes equations are governed by a set of partial differential equations (PDEs) of the general form:

$$
\frac{D \phi}{D t}=\boldsymbol{D}_{x}(\phi)+\boldsymbol{\omega}(\phi)
$$

where $\phi:=\left\{T, y_{j}\right\}, T$ is the temperature and $y_{j}$ is the mass fraction of the $j$-th species of a total of $N$ species, $D(\cdot) / D t$ is the total derivative operator, $\boldsymbol{D}_{\boldsymbol{x}}(\cdot)$ is the diffusion operator with respect to the independent space variables $\boldsymbol{x}$, and $\boldsymbol{\omega}(\cdot)$ measures the rate of change of the state $\phi$ due to finite rate chemical kinetics.

Our analysis will proceed by adopting the Lagrangian perspective to investigate how diffusion and kinetics interact so as to produce a rate of change of the state $\phi$ of a prescribed material point. The role of the vector fields of the Navier Stokes equations, namely, the action of the convective (turbulent) transport cannot be directly addressed by the present analysis, although the scalar fields are obviously subjected to their action.

Although CSP was initially developed for ordinary differential equations (ODEs) [3, 4, 28], it was later extended to partial differential equations to account for the transport aspects for application in flames $[10,11,29-32]$. The decomposition of the $N$-dimensional (local) tangent space at one space location $\boldsymbol{x}$ into a $(N-M)$-dimensional slow and a $M$-dimensional fast subspaces is obtained with reference to the set of right eigenvectors $\boldsymbol{a}(\boldsymbol{\phi})$ of the Jacobian matrix $J_{\boldsymbol{\omega}}(\phi):=(\partial \boldsymbol{\omega} / \partial \phi)$ of the source term $\boldsymbol{\omega}(\phi)$. The corresponding time scale $\tau^{j}(\phi)$, with $j=1, \ldots, N$, of the $N$-dimensional multi-scale problem is also determined as the reciprocal of the modulus of the (possibly complex) eigenvalue $\lambda^{j}(\phi)$ of the $j$-th right eigenmode $\boldsymbol{a}_{j}(\phi)$ of the Jacobian matrix, $J_{\boldsymbol{\omega}}(\phi)$, that is $\tau^{j}:=\left(\left|\lambda^{j}\right|\right)^{-1}$. For completeness, we introduce the left eigenmode $\boldsymbol{b}^{j}(\boldsymbol{\phi})$, such that $\sum_{j} \boldsymbol{a}_{j} \boldsymbol{b}^{j}=I$ and $\boldsymbol{b}^{j} \cdot \boldsymbol{a}_{k}=\delta_{k}^{j}$, with $j, k=1, \ldots, N$. Finally, the amplitude $f_{r}^{j}$ of the $j$-th mode is defined as $f_{r}^{j}:=\boldsymbol{b}^{j} \cdot \boldsymbol{\omega}$ due to the sole kinetics, and the amplitude $f_{r+d}^{j}$ of the $j$-th mode defined as $f_{r+d}^{j}:=\boldsymbol{b}^{j} \cdot\left(\boldsymbol{D}_{\boldsymbol{x}}+\boldsymbol{\omega}\right)$ due to the full RHS of Eq. (1), that is to the combined action of diffusion and kinetics.

The analytical expression of the $(N-M)$-dimensional slow manifold, referred to as slow invariant manifold (SIM), is approximated by the set of $M$ algebraic equations

$$
\boldsymbol{f}_{r}^{j}(\phi):=\boldsymbol{b}^{j}(\phi) \cdot \boldsymbol{\omega}(\phi)=0 \quad j=1, \ldots, M
$$


that express the establishment of equilibria between fast competing reactions [3, 16, 33-35]. A stable and robust criterion to declare an exhausted mode, that is one that satisfies Eq. (2), has recently been proposed in [36]. This criterion effectively determines the dimension $M$ of the fast subspace, that is of the $(N-M)$-dimensional SIM. Note that the criterion has a local character and thus, in principle, $M$ can take different values at each space location.

On the basis of the CSP decomposition, it is possible to obtain a number of metrics that allow to compare the relative contributions of one specific driving process (either diffusive or kinetic) to different aspects of the multi-scale problems. Specifically, one can list the following indices (each scaled to have unity as maximum value):

(i) the Time Scale Participation Index (TPI) measures the contribution of a specific reaction to the time scale in case of purely kinetic systems of the mode of interest [37-41];

(ii) the Amplitude Participation Index (API) measures the contribution of a process (either reaction or diffusion of a species) to the amplitude of a mode $[9,13,32,42]$ in case of reactive-diffusion systems; the API can be defined with respect to the kinetic amplitudes $\boldsymbol{f}_{r}^{j}$ or to the diffusion/kinetics amplitudes $\boldsymbol{f}_{r+d}^{j}$;

(iii) the CSP pointer $\left(\sigma_{i}^{j}:=\boldsymbol{a}_{j}^{i} \boldsymbol{b}_{i}^{j}\right)$ can be interpreted in many ways $[8,10,43,44]$; the simplest interpretation involves considering that a large value of the CSP pointer $\sigma_{i}^{j}$ implies that the dynamics of the i-th state variable proceed at a pace mostly related to the $\mathrm{j}$-th time scale.

In this work, the API index has been used for the quantification of the contribution of diffusion or kinetics [29, 32].

The dynamical analysis of the reactive flow system has been complemented by employing the tangential stretching rate (TSR) concept [23, 24], which provides an estimate of the active (slow) time scale at which the system proceeds while constrained within the SIM. The rigorous definition and underlying mathematical framework of the Tangential Stretching Rate (TSR) is detailed and discussed in previous studies [23]. Briefly, the tangential stretching rate, $\omega_{\boldsymbol{\tau}}$, is a quadratic form involving the unit vector, $\boldsymbol{\tau}$, tangential to the kinetic vector field $\boldsymbol{\omega}$ and the Jacobian of the kinetic vector field, $J_{\boldsymbol{\omega}}$, as follows:

$$
\omega_{\tau}=\tau \cdot J_{\omega} \cdot \tau
$$

It was shown in Ref. [23] that by combining the TSR definition, Eq. 3, with the eigendecomposition of the Jacobian matrix of the vector field, the TSR can be expressed as a weighted average of all the chemical eigenvalues $\lambda_{j}$ of the $j$-th mode:

$$
\omega_{r}=\sum_{j=1}^{N} W_{r}^{j} \lambda_{j}
$$

with the weight, $W_{r}^{j}$ defined as:

$$
W_{r}^{j}:=\frac{f_{r}^{j}}{|\boldsymbol{\omega}|} \sum_{k=1}^{N} \frac{f_{r}^{k}}{|\boldsymbol{\omega}|}\left(\boldsymbol{a}_{k} \cdot \boldsymbol{a}_{j}\right) .
$$

This definition, obtained with reference to a purely kinetic problem (an ODE problem), shows that the favoured time scales are those associated with eigen-modes having a large 
amplitude, and more co-linear with the vector field. It was later shown in Ref. [24] that it is possible to extend the definition to problems involving transport (a PDE problem) as follows:

$$
\omega_{r+d}=\sum_{j=1}^{N} W_{r+d}^{j} \lambda_{j},
$$

with the weight, $W_{r+d}^{j}$ defined as:

$$
W_{r+d}^{j}:=\frac{f_{r+d}^{j}}{\left|\boldsymbol{D}_{\boldsymbol{x}}+\boldsymbol{\omega}\right|} \sum_{k=1}^{N} \frac{f_{r+d}^{k}}{\left|\boldsymbol{D}_{\boldsymbol{x}}+\boldsymbol{\omega}\right|}\left(\boldsymbol{a}_{k} \cdot \boldsymbol{a}_{j}\right),
$$

where the mode amplitudes are defined as:

$$
\begin{gathered}
f_{r}^{j}:=\boldsymbol{b}^{j} \cdot \boldsymbol{\omega}, \\
f_{r+d}^{j}:=\boldsymbol{b}^{j} \cdot\left(\boldsymbol{D}_{\boldsymbol{x}}+\boldsymbol{\omega}\right) .
\end{gathered}
$$

In all the summations of the four preceding expressions, the contribution of the fast modes should be identically zero by definition of fast mode $\left(f^{k}=0\right.$ with $\left.k=1, \ldots, M\right)$. However, the numerical evaluation carried out in finite machine accuracy is not able to produce fast mode amplitudes of exactly zero amplitude. This circumstance may lead to numerical artifacts when small but non-zero fast amplitudes are multiplied by large eigenvalues. To prevent this happening, one can simply run the summation index between $\mathrm{M}+1$ to $\mathrm{N}$ without any loss of information.

The magnitude of the TSR represents the reciprocal of the system's characteristic time scale, while its positive or negative sign indicates an explosive or contractive nature of the dynamics. Hence, the TSR selects the most relevant time scales as those with the largest related amplitudes.

Furthermore, $\omega_{r}>0$ identifies explosive states where the dominant processes that tend to drive the system away from equilibrium are chemical in nature, while $\omega_{r+d}>0$ identifies explosive states where both reactions and transport processes contribute to the explosive nature of the system. When $\omega_{r} \approx \omega_{r+d}$, the contribution of transport to the dynamics of the system is negligible and the system is kinetically driven; otherwise, transport plays a non-negligible role.

The modes contributing the most to the TSR can be identified by resorting to a participation index of the $i$-th mode to the TSR, the Mode Participation Index to the TSR (TSR-MPI), defined as

$$
P_{i}^{\omega_{\tilde{\tau}}}:=\frac{W_{i}\left|\lambda_{i}\right|}{\sum_{j^{\prime}=1}^{N}\left|W_{j^{\prime}}\right| \lambda_{j^{\prime}}||} \quad \text { no summation on } i
$$

The relative contribution of each process (being a transport term or a chemical reaction) participating in the development of the system's driving timescale can be assessed with the TSR participation index, $P_{p}^{\omega_{\tilde{\tau}}}$ (TSR-API, [23-25]), that relates the $p$-th process, with $p=1,\left(2 N_{r}+N\right)$, to $\omega_{\tilde{\tau}}$ as the product of the participation index of the $i$-th mode to the TSR, $P_{i}^{\omega_{\tilde{\tau}}}$, times the API $[7,32]$ of the $p$-th process to the $i$-th mode, $P_{p}^{i}$, that is: 


$$
P_{p}^{\omega_{\tilde{\tau}}}:=\sum_{i=1}^{N} P_{i}^{\omega_{\tilde{\tau}}} \times P_{p}^{i},
$$

One can inquire where transport (either convection or diffusion or both) competes/controls/is subjected to kinetics on the basis of two derived indices $\Sigma_{r}$ and $\Sigma_{d}$ obtained by summing the absolute value of the TRS-API of all reactions and all transport processes, separately, as follows:

$$
\Sigma_{r}:=\sum_{k=1}^{2 N_{r}}\left|P_{\mathrm{r}_{k}}^{\omega_{\tilde{\tau}}}\right|,
$$

where $N_{r}$ is the number of reversible reactions in the chemical mechanism of interest, and

$$
\Sigma_{d}:=\sum_{n=1}^{N}\left|P_{\mathrm{d}_{n}}^{\omega_{\tilde{\tau}}}\right|,
$$

and by construction, it holds that $\Sigma_{d}+\Sigma_{r}=1$. In the following, $\Sigma_{d}$ and $\Sigma_{r}$ will be used in order to determine whether transport dominates over kinetics or the opposite [25], similarly to what has been introduced in the CSP context [29, 32].

All calculations of the CSP/TSR indices are carried out by employing the CSPTk package $[23,24]$, which adopts the TChem library [45] for the parsing and management of the thermokinetic data.

\section{Description of Simulation Data}

For the laminar flame used as a reference case, a steady-state solution for a freely propagating premixed $\mathrm{H}_{2}$ /air laminar flame was generated using Chemkin-Pro [46], at the pressure $\mathrm{p}_{0}=1 \mathrm{~atm}$, upstream temperature $T_{0}=300 \mathrm{~K}$ and equivalence ratio $\phi=0.7$. The detailed mechanism of Burke et al. [47] (9 species and 23 reversible reactions) was used for all simulations. For future reference, the most important reactions are listed in Table 1. In the following, the subscripts "f" and "b" are associated with forward and backward reactions, respectively.

Table 1: List of reactions providing significant contribution to the dynamics of the system.

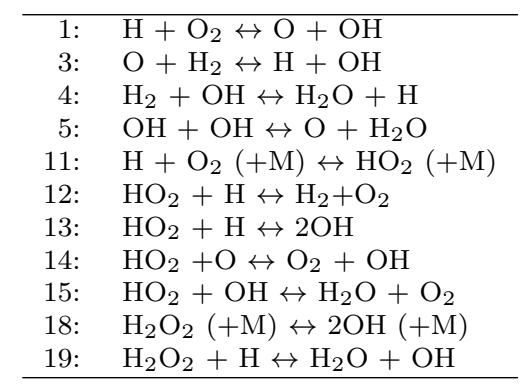

The turbulent flame simulation datasets were previously generated and detailed in [26, 27]. The S3D DNS code was utilized, which solves the fully compressible Navier-Stokes equations [48]. Spatial discretization was carried out by an eighth-order central difference scheme and an explicit fourth-order Runge-Kutta scheme was used for time marching. Each test case 
was initialized by considering a one-dimensional (1D) planar flame solution superimposed in a rectangular channel with the flow directed along the $\mathrm{x}$-axis. In the $\mathrm{x}$-axis direction, inflow and outflow boundaries are modelled using characteristic boundary conditions $[49,50]$ and all other boundaries were prescribed as periodic. The domain size is $20 \times 10 \times 10 \mathrm{~mm}^{3}$ and $8 \times 4 \times 4 \mathrm{~mm}^{3}$ for Case 2 and 4 , respectively. In both cases the domain was discretized by a uniform Cartesian grid of $512 \times 256 \times 256$ and $1280 \times 320 \times 320$ points for Case 2 and 4 , respectively. The grid spacing $(\Delta x)$ was determined based on the flame thickness $\left(\delta_{t h}\right)$. As a result, about ten grid points were ensured across the flame thickness, and the Kolmogorov length scale $(\eta)$ was bigger than the grid spacing $(\eta>1.5 \Delta x)$. A homogeneous isotropic turbulent flow field was injected at the inflow, with the mean velocity being adjusted to hold the flame within the domain for a period sufficiently long to achieve a statistically stationary solution and collect statistics.

The inflow values of normalized root-mean-square turbulent velocity fluctuation $u^{\prime} / S_{L}$, turbulent length scale to flame thickness ratio $l_{T} / \delta_{t h}$, Damköhler number Da $=\tau_{f} / \tau_{c h}=$ $\left(l_{T} / u^{\prime}\right) /\left(\delta_{t h} / S_{L}\right)$, Karlovitz number $\mathrm{Ka}=\left(\rho_{0} S_{L} \delta_{t h} / \mu_{0}\right)^{0.5}\left(u^{\prime} / S_{L}\right)^{1.5}\left(l / \delta_{t h}\right)^{-0.5}$ and turbulent Reynolds number $\operatorname{Re}_{t}=\rho_{0} u^{\prime} l_{T} / \mu_{0}$ for all cases are presented in Table 2 , where $l_{T}$ is the turbulent length scale, $S_{L}$ is the laminar flame speed, $\mu_{0}$ is the unburned gas viscosity, $\delta_{t h}=\left(T_{a d}-T_{0}\right) / \max |\nabla T|_{L}$ is the thermal flame thickness, with $\tau_{i}$ the integral eddy turnover time, and the subscript " $L$ " is used to refer to unstrained laminar flame quantities.

According to the Borghi diagram [2], the test cases marked as 2 and 4 in Table 2 fall into the thin reaction zone and distributed combustion regimes, respectively. Considering that the turbulent flow is statistically stationary (decaying in the $x$-axis direction) and the side boundary conditions are periodic, the CSP/TSR analysis is carried out at some mid-z planes, and the selected time instants are illustrative of the main features of each case. In particular, the selected physical time was $t=2.19 \mathrm{~ms}$ for Case 2 and $t=0.41 \mathrm{~ms}$ for Case 4 , at which the flames established a nearly statistically stationary condition, as has been illustrated by the time evolution of the normalized integrated heat release in Ref. [26].

Table 2: List of the inflow turbulence parameters.

\begin{tabular}{ccccccc}
\hline Case & $u^{\prime} / S_{L}$ & $l_{T} / \delta_{t h}$ & $\mathrm{Re}_{t}$ & $\mathrm{Da}$ & $\mathrm{Ka}$ & $\tau_{i}(\mu \mathrm{s})$ \\
\hline 2 & 5 & 14 & 1623 & 5.0 & 14.4 & 731 \\
4 & 14 & 4 & 1298 & 0.29 & 126 & 74.64 \\
\hline
\end{tabular}

\section{CSP Analysis of Laminar Flame}

We first present the results of the standard CSP analysis for the reference laminar flame. The evolution of selected state variables (temperature, $T$, mass fractions of $\mathrm{HO}_{2}, \mathrm{H}$, and $\mathrm{OH}$, heat release rate, $\mathrm{HRR})$ across the lean premixed $\mathrm{H}_{2}$ /air laminar flame $(p(0)=1 \mathrm{~atm}$, $T(0)=300 \mathrm{~K}, \phi=0.7)$ is shown along the $x$-axis in Fig. 1a, and along the $c_{T}$-axis in Fig. $1 \mathrm{~b}$, where $c_{T}=\left(T-T_{r}\right) /\left(T_{p}-T_{r}\right)$ is a normalized temperature, and $T_{r}$ and $T_{p}$ are the temperatures of the fresh reactants and products, respectively.

As discussed in [51], the reaction zone in hydrogen flames is composed of two parts: an Hproduction layer followed by an $\mathrm{H}$-consumption layer. In the first, the $\mathrm{HO}_{2}$ radical is produced mainly through R11f and then is rapidly depleted. Reaction R11f is fed by $\mathrm{H}$ radicals which 
Figure 1: Space evolution across the laminar flame case $(p(0)=1 \mathrm{~atm}, T(0)=300 \mathrm{~K}, \phi=0.7)$. The units of HRR is $\left[\mathrm{J} /\left(\mathrm{s} \times \mathrm{m}^{3}\right)\right]$.

are diffused from the hot downstream region. In the second layer, characterized by the consumption of $\mathrm{H}$ radicals, $\mathrm{OH}$ radicals become the dominant component of the radical pool, mainly through the chain branching reaction R1f, which becomes sufficiently active due to the increased temperature. The major exothermic reactions in the reaction zone are R11f and R4f; reaction R11f dominates the first and colder part of the reaction zone where the concentration of H-radicals increases, which is expected because the activation energy of R11f is zero. Later, the abundance of $\mathrm{OH}$ and the increased temperature favor the exothermic R4f. It is noted though that in the second layer, the highly endothermic reaction R1f becomes strong, consuming large amounts of heat. In the end, the heat release rate reaches its maximum value close to the point where $\mathrm{H}$ reaches its maximum value as well.

With reference to this description of the flame structure, and on the basis of the profiles of the selected species, of temperature, and of the heat release rate, one can infer that the reaction zone starts at $x=0.4 \mathrm{~cm}$ where $\mathrm{HO}_{2}$ starts to be produced, and can be conventionally considered to end at $x=0.5 \mathrm{~cm}$, where $\mathrm{OH}$ attains a peak value.

The spatial evolution of the kinetic time scales that characterize the local dynamics of a premixed $\mathrm{H}_{2}$ /air laminar flame $(p(0)=1 \mathrm{~atm}, T(0)=300 \mathrm{~K}, \phi=0.7)$ is shown along the $x$-axis in Fig. 2a. At each location in $x$, the number of the mode characterized by the fastest of slow timescales (i.e., the $M+1$ ) is indicated, and it varies at different locations across the flame. The time scales span a wide range of magnitude, and most of them are of dissipative nature with a related eigenvalue of negative real part (grey color in Fig. 2a). However, two of them are explosive with a related eigenvalue of positive real part (red color in Fig. 2a), with one $\left(\tau_{e, f}\right)$ faster than the other $\left(\tau_{e, s}\right)$. In the laminar flame under study, $\tau_{e, f}$ develops only in the region where temperature exhibits large variations, in contrast to the auto-ignition cases [42] where a positive eigenvalue may exist even during the isothermal induction regime.

Figure 2: Space evolution of the kinetic time scales along with temperature (a) and mode amplitudes (b), in the laminar flame case $(p(0)=1 \mathrm{~atm}, T(0)=300 \mathrm{~K}, \phi=0.7)$. (Left) Explosive/dissipative time scales are in red/grey color and orange diamonds denote the $(M+1)$-th time scale. (Right) Amplitude of mode 6 corresponds to $\tau_{e, f}$ and of mode 7 to $\tau_{e, s}$.

Figure $2 \mathrm{~b}$ shows the spatial evolution of the CSP mode amplitudes. The amplitudes $f_{r+d}^{e, f}$ and $f_{r+d}^{e, s}$ corresponding to mode $6\left(\tau_{e, f}\right)$ and mode $7\left(\tau_{e, s}\right)$, respectively, are among the largest ones. However, at $x \approx 0.42 \mathrm{~cm}, f_{r+d}^{e, s} \gg f_{r+d}^{e, f}$, suggesting that the slow explosive mode prevails over the fast explosive mode, while at the time when two time scales $\left(\tau_{e, f}\right.$ and $\left.\tau_{e, s}\right)$ merge, the amplitudes of the two explosive modes become of comparable magnitude, that is, $f_{r+d}^{e, s} \approx f_{r+d}^{e, f}$. Moreover, at the merging point, the amplitude of the fifth mode $(M=4$ at this location) increases significantly and becomes comparable to that of the two explosive modes. On the other hand, the amplitude of the $(M+1)$-th mode, the fastest active mode, is negligibly small except near the merging point, contrasting the common notion that this is expected to be most important time scale driving the system dynamics.

Figure 2 demonstrates that the fast explosive mode does not always play the role of driving the system dynamics, since its amplitude can be small. These findings indicate that 
the selection of the characteristic/driving mode/scale is not necessarily an obvious task. Alternatively, a better approach is to define the characteristic/driving mode/scale as the one with the largest amplitude. This information is provided by the TSR analysis, which unambiguously identifies the modes/scales most contributing to the slow dynamics of the system.

Figure 3: The evolution of the time scales along with temperature in the laminar flame case $(p(0)=$ $1 \mathrm{~atm}, T(0)=300 \mathrm{~K}, \phi=0.7)$, on the basis of the full and the truncated Jacobian. Grey and black colors denote the time scales of the full and truncated Jacobians, respectively, while red color represents the explosive time scales of the full Jacobian.

The time scales in Fig. 2a were computed from the eigenvalues of the $(N+1) \times(N+1)$ Jacobian of the chemical source terms, which includes both the species and temperature equations. To isolate the dependence of the system's time scales on the temperature, the time scales of the truncated $N \times N$ Jacobian of the species equations were also computed and compared with those computed on the basis of the full Jacobian. This approach has been used in order to determine the chemical and thermal runaway regimes [38, 39, 42]. Figure 3 shows the evolution of the time scales based on the truncated Jacobian, where no explosive time scale exists. This implies that the two explosive time scales obtained from the full Jacobian depend highly on temperature, therefore they are found only by accounting for the coupling of temperature and species.

\section{TSR Analysis of Laminar Flame}

An investigation of the laminar flame structure is now conducted by the TSR analysis. Considering its large dynamic range, the reactive and reactive-diffusive TSR values as well as the associated eigenvalues are reported in the logarithmic scale as follows:

$$
\Omega_{r}=\operatorname{sign}\left(\omega_{r}\right) \cdot \log _{10}\left|\omega_{r}\right|, \quad \Omega_{r+d}=\operatorname{sign}\left(\omega_{r+d}\right) \cdot \log _{10}\left|\omega_{r+d}\right|, \quad \Lambda_{i}=\operatorname{sign}\left(\lambda_{i}\right) \cdot \log _{10}\left|\lambda_{i}\right|,
$$

and, to avoid confusion, all values of $\omega, \lambda_{i}<1$ are not shown, since eigenvalues and TSR magnitudes less than unity have no meaningful impact on the system, and can be ignored. In this way, positive and negative values of $\Omega$ and $\Lambda$ denote explosive and dissipative behavior. Furthermore, all physical time scales are normalized by a nominal time of 1 second, such that the magnitudes shown in the results are nondimensional.

The above quantities have been evaluated for the reference laminar flame at $p(0)=1$ atm, $T(0)=300 \mathrm{~K}, \phi=0.7$, and plotted against the physical space $x$ (Fig. 4a) and the normalized temperature $c_{T}$ (Fig. 4b). Based on their distinct physical characteristics, the flame structures are divided by lines $\mathrm{A}-\mathrm{F}$, and referred to as the upstream zone (ahead of A), the preheat zone (A-C), the reaction zone (C-D), and the downstream zone (D-F). The boundaries of different zones were based on the zero-crossing of TSR variables. For detailed TSR analysis, zones A-C and D-F will be further divided later. It is noted that the flame thickness using the conventional formulation $\delta_{t h}=\left(T_{a d}-T_{0}\right) / \max |\nabla T|_{L}$ gives $\delta_{t h}=0.3541$ $\mathrm{mm}$, which is comparable but larger than $\delta_{t h}=0.293 \mathrm{~mm}$, based on the zero crossing of $\Omega_{r+d}$. On the other hand, based on the zero crossing of $\Omega_{r}$, the reaction zone (or Zel'dovich) thickness is $\delta_{F}=0.07 \mathrm{~mm}$, which is comparable but larger than $\delta_{F}=\alpha / s_{L}=0.03 \mathrm{~mm}$, where $\alpha$ is the mixture's thermal diffusivity. 
Figure 4: The evolution of $\Omega_{r}, \Omega_{r+d}, \Lambda_{i}$ against physical space (a) and normalized temperature $c_{T}$ (b), for the laminar flame case $(p(0)=1 \mathrm{~atm}, T(0)=300 \mathrm{~K}, \phi=0.7)$.

Within the upstream zone, both $\Omega_{r+d}$ and $\Omega_{r}$ are vanishingly small until $x=0.39 \mathrm{~cm}$, after which $\Omega_{r}$ becomes negative and increases in magnitude, suggesting that chemical activity is slowly taking place. The fact that $\Omega_{r+d}$ remains small in the same region suggests that the chemical reaction is balanced by diffusion.

The preheat zone (A-C) is marked by the first appearance of positive $\Omega_{r+d}$. In this region, $\Omega_{r}$ is large and negative, balanced by large positive values of $\Omega_{r+d}$. With reference to Eq. (4) and (5), the negative value of $\Omega_{r}$ is attributed to the development of a large magnitude of the mode amplitude $f_{r}^{j-}:=\boldsymbol{b}^{j-} \cdot \boldsymbol{\omega}$ of a mode $j$ - related to an eigenvalue $\lambda_{j-}$ with a negative real part, whereas the positive value of $\Omega_{r+d}$ arises from the development of a large magnitude of the mode amplitude $f_{r+d}^{j+}:=\boldsymbol{b}^{j+} \cdot\left(\boldsymbol{D}_{\boldsymbol{x}}+\boldsymbol{\omega}\right)$ of a mode $j+$ with a positive real part of the eigenvalue. As such, the preheat zone is characterized by the significant role of heat and mass diffusion that competes with fast contractive kinetics, such as chain termination or endothermic reactions.

In the reaction zone (C-D), marked by two crossings of $\Omega_{r}$, shows that both $\Omega_{r+d}$ and $\Omega_{r}$ are positive and close to each other in magnitude, implying that reactions are the dominant driver for explosive dynamics.

Another point to note is that within the preheat zone (A-C), $\Omega_{r+d}$ nearly coincides with the fast explosive eigenvalue $\left(\Lambda_{e, f}\right)$ (the grey line of $\Lambda_{e, f}$ exactly coincides with the $\Omega_{r+d}$ line in the earlier part of zone A-C), while in the reaction zone (C-D) $\Omega_{r+d}$ obtains values intermediate between $\Lambda_{e, f}$ and $\Lambda_{e, s}$.

Finally, the downstream region (D-F) is characterized by $\Omega_{r+d}, \Omega_{r}<0$, representing the evolution towards equilibrium within a slow (kinetic) manifold.

Note that Fig. 4 clearly demonstrates the distinct utility of TSR metrics compared to the standard explosive mode analysis. If we followed $\Lambda_{e, f}$, it would identify the overall preheat and reaction zones altogether, with the end of the reaction zone marked by the merging of the fast and slow explosive modes[42]. On the other hand, the TSR analysis clearly identifies the distinct reactive-diffusive dynamics between the preheat and reaction zones. Such features of TSR will be found valuable in the analysis of turbulent flames as will be discussed later.

Traditionally, the partitioning of the flame structure is carried out by considering other criteria based on different observables, such as the heat release rate, first or higher order derivatives of the temperature, specific species known to be effective markers for the flame structure. For the benefit of the interested reader and to establish a direct comparison among different criteria and observables, Fig. 5 shows the profiles of various observables: the heat release rate $(\mathrm{HRR})$, the temperature gradient $(\nabla T)$, and the Laplacian of temperature $\left(\nabla^{2} T\right)$, overlaid with the TSR variables. From this comparison, it is evident that there is a good agreement between the location of peak HRR and the front end of the reaction zone $\Omega_{r}=0$. On the other hand, there is no obvious correspondence between the location of peak HRR with special values of either $\nabla T$ or $\nabla^{2} T$, although the peak value of $\nabla^{2} T$ corresponds accurately to the peak of $\mathrm{HO}_{2}$ (see also Fig. 1).

Now that the net explosive/dissipative dynamical characteristics across the flame are identified, we examine the individual controlling processes that are driving the dynamics. For this purpose, Fig. 6 shows the two indices of TSR-API, $\Sigma_{r}$ and $\Sigma_{d}$, according to Eq. (12) 
Figure 5: Evolution of $\operatorname{HRR}\left(\mathrm{J} /\left(\mathrm{s} \times \mathrm{m}^{3}\right)\right), \nabla T(\mathrm{~T} / \mathrm{m}), \nabla^{2} T\left(\mathrm{~T}^{2} / \mathrm{m}^{2}\right)$ on the left axis and $\Omega_{r+d}, \Omega_{r}$ on the right axis as a function of temperature $c_{T}$. The values of HRR have been multiplied by $8 \times 10^{6}$ and the values of $\nabla T$ have been multiplied by $10^{6}$.

and Eq. (13), respectively. Since $\Sigma_{d}+\Sigma_{r}=1$, we can identify the diffusion-controlled $\left(\Sigma_{d}>\Sigma_{r}\right)$ and reaction-controlled $\left(\Sigma_{d}<\Sigma_{r}\right)$ regions. Fig. 6 reveals that even within the preheat zone (A-C), where $\Omega_{r+d}>0$, the cause of the explosive nature comes from diffusion in further upstream and then from reactions. Likewise, in the downstream region the dissipative dynamics are governed initially by chemical and then by diffusive equilibration processes. These crossing points in the preheat and downstream zones are denoted as lines B and E, respectively.

Figure 6: Spatial profiles of $\Omega_{r}$ and $\Omega_{r+d}$ (left axis) and TSR-API $\Sigma_{r}$ and $\Sigma_{d}$ (right axis), for laminar flame $(p(0)=1 \mathrm{~atm}, T(0)=300 \mathrm{~K}, \phi=0.7)$.

Figure 7: Spatial profiles of the largest TSR-API contributions from diffusive (left) and kinetic (right) processes, for the laminar flame $(p(0)=1 \mathrm{~atm}, T(0)=300 \mathrm{~K}, \phi=0.7)$.

Subsequently, specific processes contributing the most to the explosive/dissipative dynamics in each region can be assessed by inspecting the budget analysis of TSR-API as shown in Fig. 7. To avoid the clutter, contributions from diffusive (the budget of $\Sigma_{d}$ ) and the contributions from reactive (the budget of $\Sigma_{r}$ ) are separately shown. Some physical insights are drawn from the results as follows.

(i) Region A-B: the explosive dynamics are mainly driven by the diffusion of heat and fast species like $\mathrm{H}$ and $\mathrm{H}_{2}$, while the recombination reaction $\mathrm{R} 11 \mathrm{f}$ is the primary source of dissipation.

(ii) Region B-C: the explosive dynamics are mainly driven by the chemical pathways, R11f, R4f, R3f, and R1f (in the order of magnitude). As it is well known for hydrogen/oxygen chemistry, at this low temperature $(<1000 \mathrm{~K})$ even the recombination reaction $\mathrm{R} 11 \mathrm{f}$ leads to subsequent branching, and hence drives the explosive dynamics.

(iii) Region C-D: the explosive dynamics are driven by both reaction and diffusion. As for the reaction, the same reactions identified in region B-C are responsible. Note that the temperature is still low in this region, and R11f is the primary heat releasing reaction in the hydrogen/air flames, thus the recombination reaction R11f still contributes to explosive dynamics. As for the diffusion, in this region of strong gradients, the diffusion of $\mathrm{H}_{2}$ (gain) wins over the diffusion of heat and $\mathrm{H}$ (loss) toward the explosive dynamics.

(iv) Region D-E: the dissipative dynamics are driven by both diffusion and reaction, but diffusion of reactants serve as the dominant cause of losses.

(v) Region E-F: the dissipative dynamics are driven by both diffusion and reaction, but at this region of low concentration gradient, the reactions play a dominant role for the dissipation. 


\section{TSR Analysis of Turbulent Flames}

In this section, TSR analysis is applied to turbulent flames at two different combustion regimes and the characteristics are compared with those of the laminar flames. For each turbulent flame simulation case, an instantaneous solution field is taken as discussed in Section 3, for which the data of a mid-z plane were processed. Note that a few different planes were also analyzed and consistent results were found. A sample of the analysis of three additional time steps is included as Supplementary Material.

The two-dimensional (2D) mass fraction fields of selected species on the z-planes are shown in Fig. 8, namely, the spatial evolution of the mass fraction of $\mathrm{H}, \mathrm{HO}_{2}$ and $\mathrm{OH}$ for Case 2 (upper row) and Case 4 (lower row). Based on the typical laminar flame structure, these species approximately represent the preheat, main reaction, and downstream regions, respectively. Comparing the $\mathrm{HO}_{2}$ fields for Cases 2 and 4 clearly underlines the main difference of the thin reaction zone regime in contrast to the distributed combustion regime. In the thin reaction zone regime, $\mathrm{HO}_{2}$ is mostly confined in a well connected thin region, indicating that the basic laminar flame structure is intact. In contrast, in Case 4 the action of small scale turbulence creates complex structures in the preheat zone, while $\mathrm{H}$ isocontours exhibit some pockets ahead of the main reaction zone. The $\mathrm{OH}$ layers, located further downstream, appear to be broader but without much turbulence action due to the flow laminarization by heat release. These species have been commonly used in the literature as flame front markers. The results show that the flame front identification yields significantly different behavior depending on which species is used. It is also noted that unlike $\mathrm{HO}_{2}$, $\mathrm{H}$ and $\mathrm{OH}$ obtain significantly lower maximum values in Case 4 compared to Case 2, thus further complicating their use as universal flame front markers.

Figure 8: The spatial distribution of mass fraction of $(\mathrm{a}, \mathrm{d}) \mathrm{H},(\mathrm{b}, \mathrm{e}) \mathrm{HO}_{2}$ and $(\mathrm{c}, \mathrm{f}) \mathrm{OH}$ for Case 2 (upper row) and Case 4 (lower row).

Using the TSR analysis, Fig. 9 shows the distributions of $\Omega_{r+d}, \Omega_{r}$ and $\Lambda_{e, f}$ in physical space for both cases. As discussed in the laminar flame case, it is evident that the region of $\Omega_{r+d}>0$ differs significantly from the region of $\Lambda_{e, f}$ existence; there is a wide region where $\Lambda_{e, f}$ indicates explosive dynamics but $\Omega_{r+d}$ shows dissipative. This tendency is more pronounced in Case 2, indicating stronger interaction between kinetics and transport. Finally, the region of $\Omega_{r}>0$ adequately represents the major heat release region as will be discussed next.

Figure 9: The spatial distribution of (a,d) $\Omega_{r+d}$, (b,e) $\Omega_{r}$ and (c,f) $\Lambda_{e, f}$ for Cases 2 (upper row) and 4 (lower row).

To assess which metric serves as a proper flame marker, the spatial distributions of temperature and the heat release rate are plotted in Fig. 10, in which the contour lines of $\Omega_{r}=0$ (white solid lines) and $\Lambda_{e, f}=0$ contour lines (black solid lines) are overlaid, for both Cases 2 and 4. Contrary to $\Lambda_{e, f}$, the region of $\Omega_{r}>0$ is confined in a thin layer and aligns well with the region of high heat release rate. In contrast, the region of $\Lambda_{e, f}$ is much wider, covering a significantly larger area which is identified as dissipative by the TSR analysis. Again, the message is that the positive eigenvalue alone does not guarantee 
explosive dynamics; the TSR metric combines the positive eigenvalues weighted with their amplitudes, thus providing a more accurate description of explosive dynamics in the system.

Comparing Figs. 9 and 10, it is evident that the deviation between $\Omega_{r+d}$ and $\Lambda_{e, f}$ relates mainly to the preheat zone. This is a notable difference between Case 2 and the laminar flame, where $\Omega_{r+d}$ was shown to track $\Lambda_{e, f}$ in the preheat zone. These findings suggest that the different turbulent conditions have a qualitatively different impact on the preheat zone. In the thin reaction zone case, the explosive dynamics are suppressed, while at higher Ka the explosive dynamics tend to resemble the profile of a laminar one in the sense that the preheat zone is mainly characterized by explosive dynamics.

Figure 10: The spatial distribution of $(\mathrm{a}, \mathrm{c})$ temperature $(\mathrm{K})$ and $(\mathrm{b}, \mathrm{d})$ heat release rate overlaid with the $\Omega_{r}=0$ (white solid lines) and $\Lambda_{e, f}=0$ contour lines (black solid lines), for Case 2 (upper row) and Case 4 (lower row).

The topological characterization of the reactive flow by means of the TSR indices is rendered with the three-dimensional (3D) plot shown in Fig. 11, where the height of each scatter plot at each $(\mathrm{x}, \mathrm{y})$ location of the domain is proportional to the magnitude of $\Omega_{r}$, while the color legend is associated with the value of HRR. By construction, a scatter point on the upper half of the 3D plot corresponds to an explosive situation, while on the lower half to a contractive/dissipative situation. The dark red color on the scatter points implies that there exists a positive correlation between $\Omega_{r}$ and HRR, that is, $\Omega_{r}$ and HRR attain high values at the same location. Clearly, Fig. 11 suggests that the heat release rate is well correlated with $\Omega_{r}$ (as indicated by the laminar test case, see Fig. 5).

Figure 11: The height of each scatter plot is proportional to the magnitude of $\Omega_{r}$, while the color legend is associated with the value of HRR; Left (a) is for Case 2; Right (b) is for Case 4. In both cases, the values of HRR have been normalized by their maximum values.

Figure 12: Scatter plots of $\Omega_{r}$ (a), $\Omega_{r+d}$ (b) and $\Lambda_{e, f}$ (c) against $c_{T}$, for laminar (red), thin reaction zone regime (Case 2 in blue) and distributed combustion regime (Case 4 in green). The conditional averages of $\Omega_{r}$ (left) and $\Omega_{r+d}$ for Case 2 and 4 are overlaid with cyan-dashed and black-solid lines, respectively.

Fig. 12 shows the scatter plot of $\Omega_{r+d}, \Omega_{r}$ and $\Lambda_{e, f}$ as a function of $c_{T}$, along with their conditional averages. To avoid clutters, the scatter points were taken from the $2 \mathrm{D}$ plane, but the conditional averages were calculated from the entire three-dimensional domain. It is seen that data points for $\Omega_{r}$ for Case 2 (thin reaction zone regime) show a much lower level of scatters compared to that for Case 4 (distributed regime), suggesting that a higher level of turbulence results in larger fluctuations. Nevertheless, the conditional averages nearly coincide with the laminar flame values for both cases, again suggesting that $\Omega_{r}$ serves as a good marker to identify the primary reaction zones even in high Ka conditions.

On the other hand, the profile of $\Omega_{r+d}$ appears to be qualitatively different in the preheat zone between Case 2 and the laminar flame, since in the early part of the preheat zone (i.e., $\left.c_{T}<0.07\right) \Omega_{r+d}$ is explosive in the laminar flame and dissipative in Case 2, as was also shown in Fig. 9. Rather, Case 4 recovers the profile closer to the laminar flame since $\Omega_{r+d}$ is 
mainly explosive in that region. This behavior is also consistently shown in the conditional averages (lines). Thus, it appears that turbulence affects different cases in a different manner, resulting in such unexpected non-monotonic behavior. A possible explanation is that in the low Ka case, the integral-scale eddies are large enough to interact with the preheat zone, leaving the much smaller reaction zone unaffected. As a result of the strong interaction of the turbulent eddies with the preheat zone, the local dynamics become dissipative. On the other hand, in the higher Ka case, the turbulent kinetic energy is higher yet the energy dissipation is much larger, as compared to the lower Ka case. As a result, the dynamics return to that of the laminar flame, with the exception of the reaction zone which is broadened due to the intense interaction of the small-scale eddies with the size-comparable reaction zone.

The observed non-monotonic flame response to turbulence is an unexpected finding and needs further investigation examining cases for different turbulent conditions. In both cases, in the region where $\Omega_{r+d}>0$, turbulence creates fluctuations to yield the local values of $\Omega_{r+d}$ that can be either positive or negative. A similar finding was reported in Ref. [25] in the context of the comparative investigation of two turbulent flames, in the corrugated flamelet and thin reaction zone combustion regimes. The present analysis shows that this behavior is more pronounced in Case 4 compared to Case 2. Obviously, the increased dispersion that characterizes $\Omega_{r+d}$ in Case 4 is the result of the increased turbulent activity.

In contrast to the behavior of the TSR metrics, the profile of $\Lambda_{e, f}$ appears to be largely insensitive to the different turbulent conditions, with both turbulent cases nearly collapsing the laminar results with minor level of scatters. Again, this demonstrates the fact that the positive eigenvalue alone is not adequate in capturing the detailed turbulence effect on the net explosive dynamics in the system.

To further highlight the difference between the explosive mode and TSR diagnostics, Fig. 13 compares the regions of $\Lambda_{e, f}>0$ and $\Omega_{r+d}>0$, colored in green and red, respectively, for Cases 2 and 4 . The isolines of $\Omega_{r}=0$ are overlaid with black solid line. Case 2 shows that $\Lambda_{e, f}$ tends to prematurely predict explosive dynamics, while the TSR accounting for the amplitudes of all modes predicts a much lower level of explosive behavior. This tendency is reduced at higher $\mathrm{Ka}$ (Case 4), where the differences become smaller as a wide range of turbulent preheat zones become diffusively explosive. While the pictures appear similar in Case 4, the eigenvalue diagnostics would have been interpreted as reaction zone broadening, while the TSR diagnostics correctly describes this region as diffusion-dominant region.

Figure 13: The contour lines of $\Omega_{r+d}>0$ (in red) and $\Lambda_{e, f}$ (green) overlaid with the $\Omega_{r}=0$ isoline (black), for Case 2 (a) and Case 4 (b).

Figure 14: The distribution of $\Sigma_{r}>50 \%$ (red) and $\Sigma_{d}>50 \%$ (green) overlaid with the contour lines of $\Omega_{r+d}>0$ (in black), for Case 2 (a) and Case 4 (b). The darkness of each color is representative of the intensity of the relevant quantity.

The light green areas in Fig. 13 represent the regions where explosive modes $\left(\Lambda_{e, f}\right)$ exist but the net behavior is dissipative. To identify which processes are causing the dissipation, Fig. 14 shows the regions of $\Omega_{r+d}<0$ in green and red color map, representing that dissipation is dominated by reactions $\left(\Sigma_{r}>0.5\right)$ and diffusion $\left(\Sigma_{d}>0.5\right)$, respectively. The regions of $\Omega_{r+d}>0$ are blacked out. In Case 2, the dissipative behavior in the $\Lambda_{e, f}>0$ zone is mainly due to the reactions, while in Case 4 both chemistry and diffusion play significant 
role. In summary, relative to the laminar flames where the preheat zone is dominated by the diffusion-reaction-driven explosive dynamics (e.g., zone A-C in Fig. 6), turbulent eddies suppress the explosive behavior in the preheat zone. In the thin reaction zone regime, this is caused by the suppression of the reactive processes, while in the distributed combustion regime turbulent dissipation plays the dominant role. The results manifest the complex dynamics of the turbulence-chemistry interaction in turbulent flames at different Karlovitz numbers, which need further investigation.

\section{Conclusions}

The topology and structure of turbulent hydrogen/air premixed flames at different Karlovitz number conditions were investigated by the computational singular perturbation (CSP) method with extensive use of the tangential stretching rate (TSR) analysis. The standard CSP allows an automated identification of the local time scales of the multi-scale problems with a large number of degrees of freedom, decomposed into slow and fast components. In addition, TSR allows to identify the most energetic time scale during both the explosive and dissipative regimes. Taken together, the method serves as an unambiguous identification of important regions in turbulent flames, even at high Karlovitz number conditions, where traditional metrics yield incorrect descriptions or require case-by-case adjustments of the definitions.

The analytical tools were first applied to a basic laminar flame, which revealed a systematic description about the dynamical structure of a laminar flame. Additional index metrics further allow identifications of important chemical and transport processes that are driving the explosive characteristics. One key message from this study is that TSR provides a more comprehensive and correct description of the reactive system dynamics, while the method based solely on the eigenvalues may lead to misleading interpretation of physics due to the neglect of the corresponding mode amplitudes.

For turbulent flames, it was generally found that the reactive TSR $\left(\Omega_{r}\right)$ properly identifies the main reaction zones, while the reactive-diffusive TSR $\left(\Omega_{r+d}\right)$ depicts the broadened preheat zones where diffusion process drives the explosive dynamics. However, the detailed sub-processes responsible for the explosive dynamics in the preheat zone depend on the specific turbulence conditions, and the net effect was sometimes found to be nonmonotonic. Further analysis with a larger number of simulation cases is required to accomplish a complete understanding. This includes analysis of high Ka turbulent flames in different configurations, such as jet flames $[52,53]$, which reported some distinct flame characteristics.

Finally, we remark that the TSR analysis is compatible with a Lagrangian framework. By combining it with a flame particle tracking approach [54], the analysis can unravel important information about the dynamical evolution of turbulence-flame interaction without an introduction of ad hoc diagnostic metrics. Further work is needed to explore this approach.

\section{Acknowledgements}

This work was sponsored by competitive research funding from King Abdullah University of Science and Technology (KAUST). MV acknowledges the support of the Italian Ministry of University and Research (MIUR). 


\section{References}

[1] H. G. Im, Direct Numerical Simulations for Combustion Science: Past, Present, and Future. in: De S., Agarwal A., Chaudhuri S., Sen S. (eds) Modeling and Simulation of Turbulent Combustion, Energy, Environment, and Sustainability, Springer, 2018.

[2] N. Peters, Turbulent Combustion, Cambridge Monographs on Mechanics, Cambridge University Press, 2000.

[3] S. Lam, D. Goussis, Understanding complex chemical kinetics with computational singular perturbation, Proc. Combust. Inst. 22 (1) (1989) 931-941.

[4] S. Lam, D. Goussis, The CSP method for simplifying kinetics, Int. J. Chem. Kinet. 26 (4) (1994) $461-486$.

[5] D. A. Goussis, M. Valorani, An efficient iterative algorithm for the approximation of the fast and slow dynamics of stiff systems, J. Comput. Phys. 214 (1) (2006) 316-346.

[6] S. Gupta, H. G. Im, M. Valorani, Classification of ignition regimes in HCCI combustion using computational singular perturbation, Proc. Combust. Inst. 33 (2) (2011) 2991-2999.

[7] S. Gupta, H. G. Im, M. Valorani, Analysis of n-heptane auto-ignition characteristics using computational singular perturbation, Proc. Combust. Inst. 34 (1) (2013) 1125 - 1133.

[8] E. A. Tingas, D. C. Kyritsis, D. A. Goussis, Autoignition dynamics of DME/air and EtOH/air homogeneous mixtures, Combust. Flame 162 (9) (2015) 3263-3276.

[9] D. M. Manias, E. A. Tingas, C. E. Frouzakis, K. Boulouchos, D. A. Goussis, The mechanism by which $\mathrm{CH}_{2} \mathrm{O}$ and $\mathrm{H}_{2} \mathrm{O}_{2}$ additives affect the autoignition of $\mathrm{CH}_{4}$ /air mixtures, Combust. Flame 164 (2016) $111-125$.

[10] M. Valorani, H. N. Najm, D. A. Goussis, CSP analysis of a transient flame-vortex interaction: time scales and manifolds, Combust. Flame 134 (1) (2003) 35-53.

[11] H. N. Najm, M. Valorani, D. A. Goussis, J. Prager, Analysis of methane-air edge flame structure, Combust. Theor. Model. 14 (2) (2010) 257-294.

[12] M. Jaasim, E.-A. Tingas, F. E. Hernández Pérez, H. G. Im, Computational singular perturbation analysis of super-knock in SI engines, Fuel 225 (2018) 184-191.

[13] W. Song, E.-A. Tingas, H. G. Im, A computational analysis of methanol autoignition enhancement by dimethyl ether addition in a counterflow mixing layer, Combust. Flame 195 (2018) 84-98.

[14] M. Valorani, D. A. Goussis, F. Creta, H. N. Najm, Higher order corrections in the approximation of lowdimensional manifolds and the construction of simplified problems with the CSP method, J. Comput. Phys. 209 (2) (2005) 754-786.

[15] M. Valorani, F. Creta, D. A. Goussis, J. C. Lee, H. N. Najm, An automatic procedure for the simplification of chemical kinetic mechanisms based on CSP, Combust. Flame 146 (1) (2006) 29 - 51.

[16] E.-A. Tingas, D. J. Diamantis, D. A. Goussis, Issues arising in the construction of QSSA mechanisms: the case of reduced n-heptane/air models for premixed flames, Combust. Theor. Model. https://doi.org/10.1080/13647830.2018.1470333 (2018) 1-35.

[17] R. M. Galassi, P. P. Ciottoli, S. M. Sarathy, H. G. Im, S. Paolucci, M. Valorani, Automated chemical kinetic mechanism simplification with minimal user expertise, Combust. Flame 197 (2018) 439-448.

[18] P. P. Ciottoli, R. M. Galassi, P. E. Lapenna, G. Leccese, D. Bianchi, F. Nasuti, F. Creta, M. Valorani, Csp-based chemical kinetics mechanisms simplification strategy for non-premixed combustion: An application to hybrid rocket propulsion, Combust. Flame 186 (2017) 83-93.

[19] T. Grenga, S. Paolucci, M. Valorani, Sensitivity analysis and mechanism simplification using the Gscheme framework, Combust. Flame 189 (2018) $275-287$.

[20] M. Valorani, S. Paolucci, The G-scheme: A framework for multi-scale adaptive model reduction, J. Comput. Phys. 228 (13) (2009) $4665-4701$.

[21] M. Valorani, S. Paolucci, P. P. Ciottoli, R. M. Galassi, Entropy production and timescales, Combust. Theor. Model. 21 (1) (2017) 137-157.

[22] A. Adrover, F. Creta, M. Giona, M. Valorani, Stretching-based diagnostics and reduction of chemical kinetic models with diffusion, J. Comput. Phys. 225 (2) (2007) 1442-1471.

[23] M. Valorani, S. Paolucci, E. Martelli, T. Grenga, P. P. Ciottoli, Dynamical system analysis of ignition phenomena using the tangential stretching rate concept, Combust. Flame 162 (8) (2015) 2963-2990.

[24] M. Valorani, P. P. Ciottoli, R. M. Galassi, Tangential stretching rate (TSR) analysis of non premixed reactive flows, Proc. Combust. Inst. 36 (1) (2017) 1357-1367.

[25] D. Manias, A.-E. Tingas, F. E. Hernández Pérez, H. G. Im, R. Malpica Galassi, P. P. Ciottoli, M. Val- 
orani, Analysis of hydrogen/air turbulent premixed flames at different Karlovitz numbers using computational singular perturbation, in: 2018 AIAA Aerospace Sciences Meeting, 2018, p. 0364.

[26] H. G. Im, P. G. Arias, S. Chaudhuri, H. A. Uranakara, Direct numerical simulations of statistically stationary turbulent premixed flames, Combust. Sci. Techn. 188 (8) (2016) 1182-1198.

[27] P. Arias, H. Uranakar, S. Chaudhuri, H. Im, Direct numerical simulations of flow-chemistry interactions in statistically turbulent premixed flames, in: APS Meeting Abstracts, 2015.

[28] D. A. Goussis, M. Valorani, F. Creta, H. N. Najm, -inertial manifolds with csp, in: Computational Fluid and Solid Mechanics 2003, Elsevier, 2003, pp. 1951-1954.

[29] M. Hadjinicolaou, D. A. Goussis, Asymptotic solution of stiff PDEs with the CSP method: The reaction diffusion equation, SIAM J. Sci. Comput. 20 (3) (1998) 781-810.

[30] M. Valorani, F. Creta, D. A. Goussis, Local and global manifolds in stiff reaction-diffusion systems, in: Computational Fluid and Solid Mechanics 2003, Elsevier, 2003, pp. 1548-1551.

[31] D. A. Goussis, M. Valorani, F. Creta, H. N. Najm, Reactive and reactive-diffusive time scales in stiff reaction-diffusion systems, Prog. Comput. Fluid Dy. 5 (6) (2005) 316-326.

[32] J. Prager, H. N. Najm, M. Valorani, D. Goussis, Structure of n-heptane/air triple flames in partiallypremixed mixing layers, Combust. Flame 158 (11) (2011) 2128-2144.

[33] D. Goussis, On the construction and use of reduced chemical kinetic mechanisms produced on the basis of given algebraic relations, J. Comput. Phys. 128 (2) (1996) 261-273.

[34] A. Massias, D. Diamantis, E. Mastorakos, D. Goussis, Global reduced mechanisms for methane and hydrogen combustion with nitric oxide formation constructed with csp data, Combust. Theor. Model. 3 (2) (1999) 233-257.

[35] A. Massias, D. Diamantis, E. Mastorakos, D. Goussis, An algorithm for the construction of global reduced mechanisms with csp data, Combust. Flame 117 (4) (1999) 685-708.

[36] M. Valorani, P. P. Ciottoli, R. M. Galassi, S. Paolucci, T. Grenga, E. Martelli, Enhancements of the G-Scheme framework, Flow Turbul. Combust. (2018) 1-11.

[37] E.-A. Tingas, D. C. Kyritsis, D. A. Goussis, Comparative investigation of homogeneous autoignition of DME/air and EtOH/air mixtures at low initial temperatures, Combust. Theor. Model. 21 (1) (2017) 93-119.

[38] E. A. Tingas, D. C. Kyritsis, D. A. Goussis, Algorithmic determination of the mechanism through which $\mathrm{H}_{2} \mathrm{O}$-dilution affects autoignition dynamics and $\mathrm{NO}$ formation in $\mathrm{CH}_{4}$ /air mixtures, Fuel 183 (2016) 90-98.

[39] E. A. Tingas, H. G. Im, D. C. Kyritsis, D. A. Goussis, The use of $\mathrm{CO}_{2}$ as an additive for ignition delay and pollutant control in $\mathrm{CH}_{4}$ /air autoignition, Fuel 211 (2018) 898-905.

[40] S. M. Sarathy, E.-A. Tingas, E. F. Nasir, A. Detogni, Z. Wang, A. Farooq, H. Im, Three-stage heat release in n-heptane auto-ignition, Proc. Combust. Inst.

[41] E.-A. Tingas, Z. Wang, S. M. Sarathy, H. G. Im, D. A. Goussis, Chemical kinetic insights into the ignition dynamics of n-hexane, Combust. Flame 188 (2018) 28-40.

[42] D. J. Diamantis, E. Mastorakos, D. A. Goussis, $\mathrm{H}_{2}$ /air autoignition: The nature and interaction of the developing explosive modes, Combust. Theor. Model. 19 (3) (2015) 382-433.

[43] E. A. Tingas, D. C. Kyritsis, D. A. Goussis, Ignition delay control of DME/air and EtOH/air homogeneous autoignition with the use of various additives, Fuel 169 (2016) 15-24.

[44] E. A. Tingas, D. M. Manias, S. M. Sarathy, D. A. Goussis, $\mathrm{CH}_{4}$ /air homogeneous autoignition: A comparison of two chemical kinetics mechanisms, Fuel 223 (2018) 74-85.

[45] C. Safta, H. N. Najm, O. Knio, TChem-a software toolkit for the analysis of complex kinetic models, Sandia Report, SAND2011-3282.

[46] Reaction Design Inc., CHEMKIN-PRO, san Diego, CA (2011).

[47] M. P. Burke, M. Chaos, Y. Ju, F. L. Dryer, S. J. Klippenstein, Comprehensive H2/O2 kinetic model for high-pressure combustion, Int. J. Chem. Kinet. 44 (7) (2012) 444-474.

[48] J. H. Chen, A. Choudhary, B. De Supinski, M. DeVries, E. R. Hawkes, S. Klasky, W.-K. Liao, K.L. Ma, J. Mellor-Crummey, N. Podhorszki, et al., Terascale direct numerical simulations of turbulent combustion using s3d, Comput. Sc. \& Discov. 2 (1) (2009) 015001.

[49] B. J. Lee, C. S. Yoo, H. G. Im, Dynamics of bluff-body-stabilized premixed hydrogen/air flames in a narrow channel, Combust. Flame 162 (6) (2015) 2602-2609.

[50] C. S. Yoo, H. G. Im, Characteristic boundary conditions for simulations of compressible reacting flows with multi-dimensional, viscous and reaction effects, Combust. Theor. Model. 11 (2007) 259-286.

[51] C. K. Law, Combustion physics, Cambridge university press, 2010.

[52] H. Wang, E. R. Hawkes, J. H. Chen, B. Zhou, Z. Li, M. Aldén, Direct numerical simulations of a high 
Karlovitz number laboratory premixed jet flame-an analysis of flame stretch and flame thickening, J. Fluid Mech. 815 (2017) 511-536.

[53] H. Wang, E. R. Hawkes, B. Zhou, J. H. Chen, Z. Li, M. Aldén, A comparison between direct numerical simulation and experiment of the turbulent burning velocity-related statistics in a turbulent methane-air premixed jet flame at high Karlovitz number, Proc. Combust. Inst. 36 (2) (2017) 2045-2053.

[54] H. A. Uranakara, S. Chaudhuri, H. L. Dave, P. G. Arias, H. G. Im, A flame particle tracking analysis of turbulence-chemistry interaction in hydrogen-air premixed flames, Combust. Flame 163 (2016) 220240. 


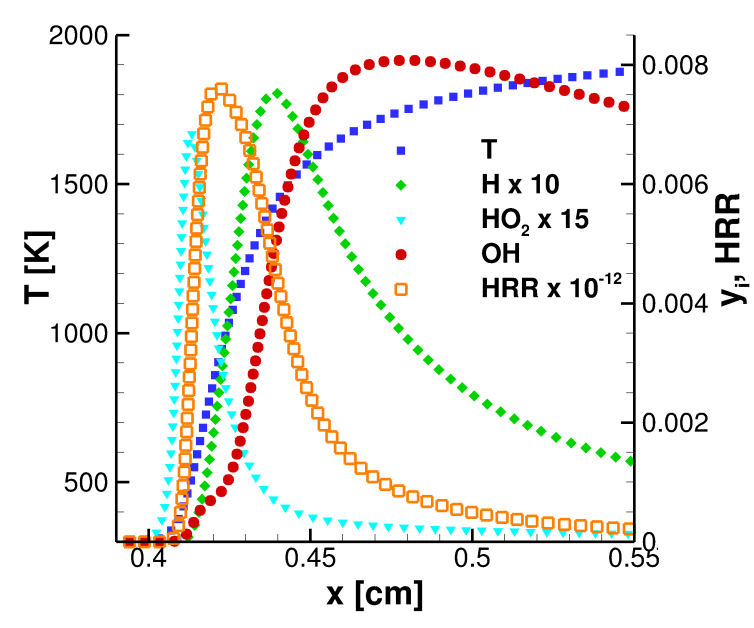

(a)

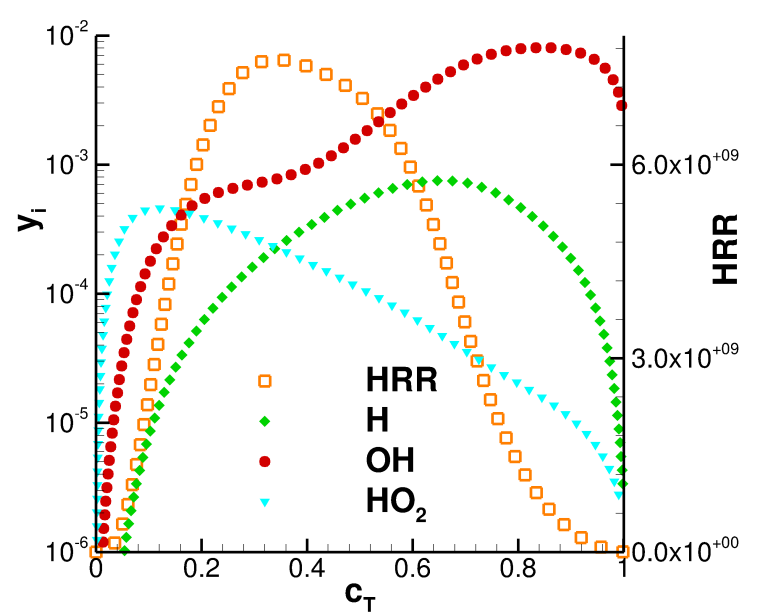

(b)

Figure 1: Space evolution across the laminar flame case $(p(0)=1 \mathrm{~atm}, T(0)=300 \mathrm{~K}, \phi=0.7)$. The units of HRR is $\left[\mathrm{J} /\left(\mathrm{s} \times \mathrm{m}^{3}\right)\right]$.

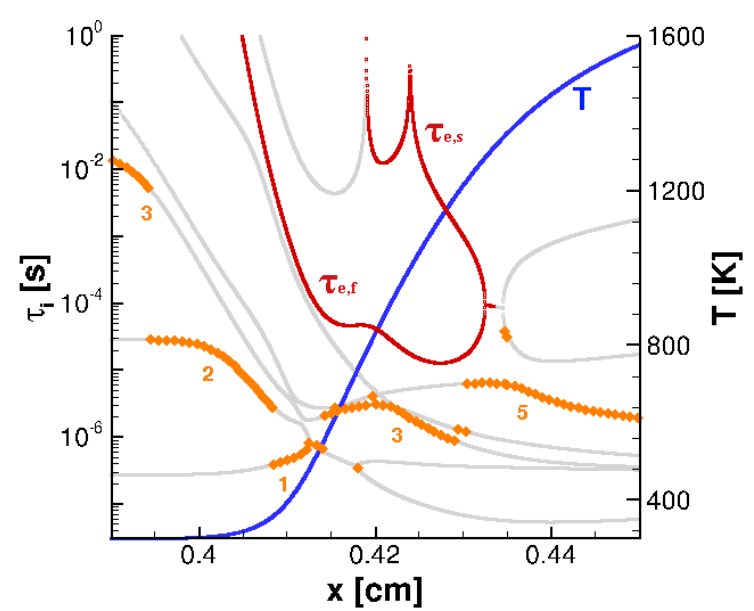

(a)

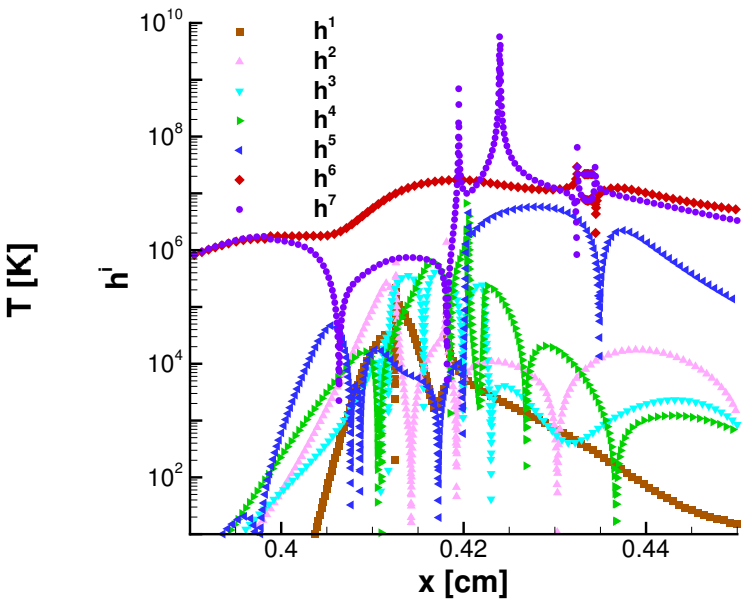

(b)

Figure 2: Space evolution of the kinetic time scales along with temperature (a) and mode amplitudes (b), in the laminar flame case $(p(0)=1 \mathrm{~atm}, T(0)=300 \mathrm{~K}, \phi=0.7)$. (Left) Explosive/dissipative time scales are in red/grey color and orange diamonds denote the $(M+1)$-th time scale. (Right) Amplitude of mode 6 corresponds to $\tau_{e, f}$ and of mode 7 to $\tau_{e, s}$. 


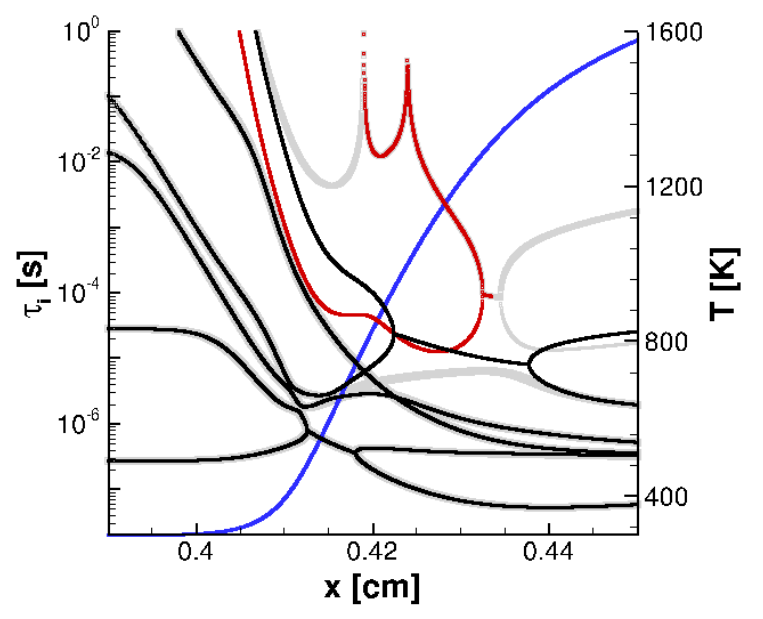

Figure 3: The evolution of the time scales along with temperature in the laminar flame case $(p(0)=$ $1 \mathrm{~atm}, T(0)=300 \mathrm{~K}, \phi=0.7)$, on the basis of the full and the truncated Jacobian. Grey and black colors denote the time scales of the full and truncated Jacobians, respectively, while red color represents the explosive time scales of the full Jacobian.

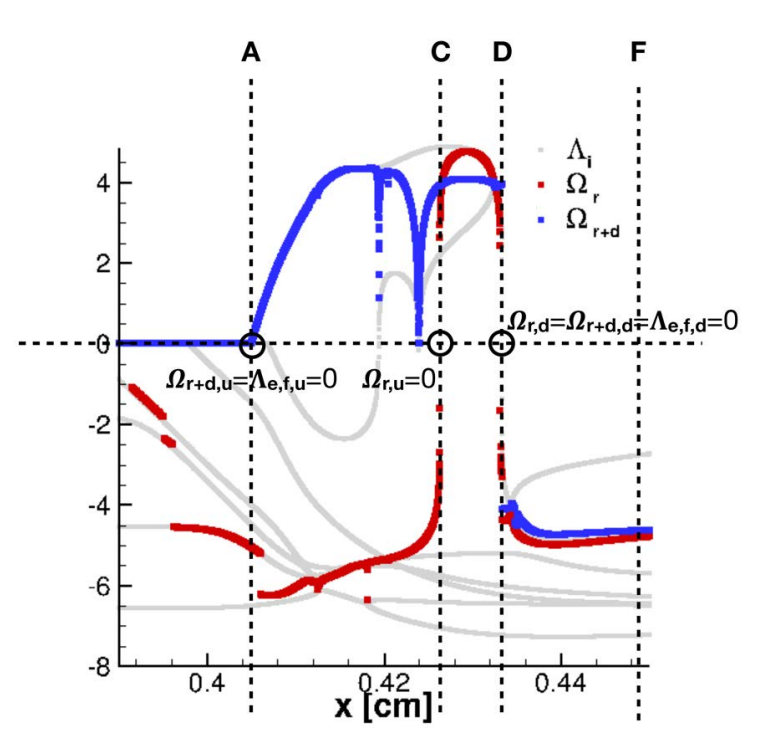

(a)

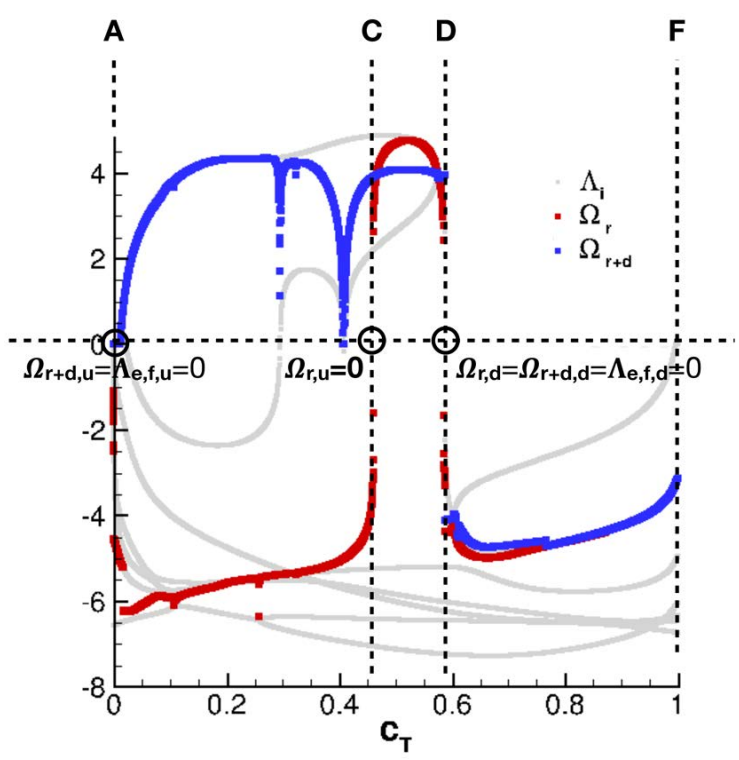

(b)

Figure 4: The evolution of $\Omega_{r}, \Omega_{r+d}, \Lambda_{i}$ against physical space (a) and normalized temperature $c_{T}$ (b), for the laminar flame case $(p(0)=1 \mathrm{~atm}, T(0)=300 \mathrm{~K}, \phi=0.7)$. 


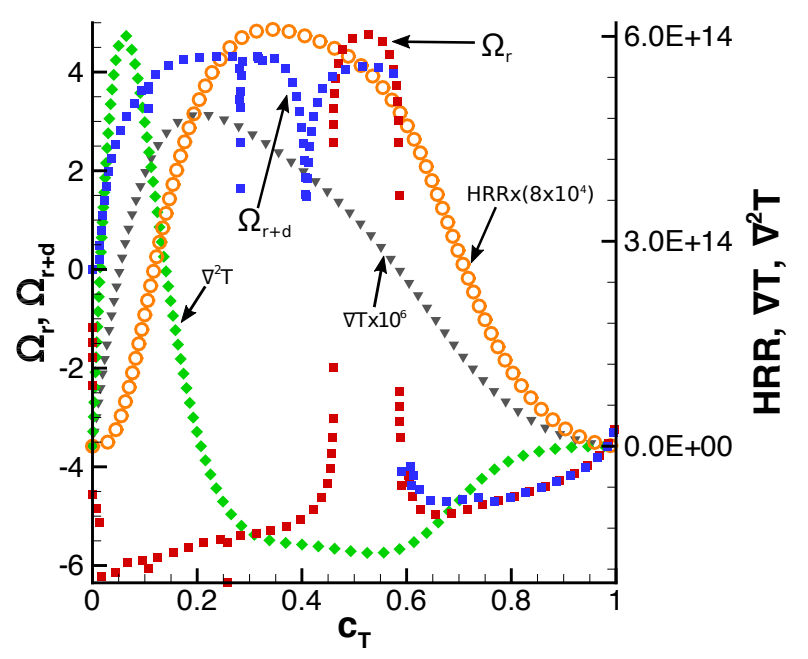

Figure 5: Evolution of $\operatorname{HRR}\left(\mathrm{J} /\left(\mathrm{s} \times \mathrm{m}^{3}\right)\right), \nabla T(\mathrm{~T} / \mathrm{m}), \nabla^{2} T\left(\mathrm{~T}^{2} / \mathrm{m}^{2}\right)$ on the left axis and $\Omega_{r+d}, \Omega_{r}$ on the right axis as a function of temperature $c_{T}$. The values of HRR have been multiplied by $8 \times 10^{6}$ and the values of $\nabla T$ have been multiplied by $10^{6}$.

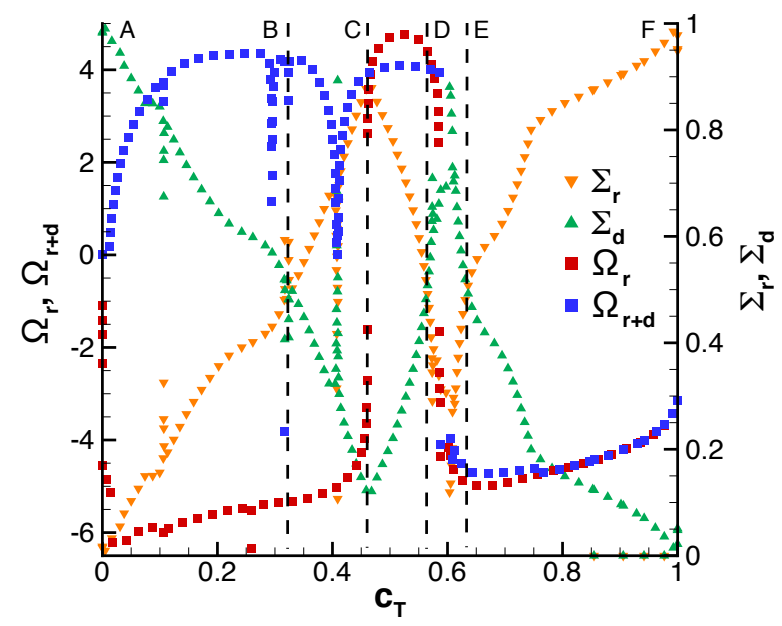

Figure 6: Spatial profiles of $\Omega_{r}$ and $\Omega_{r+d}$ (left axis) and TSR-API $\Sigma_{r}$ and $\Sigma_{d}$ (right axis), for laminar flame $(p(0)=1 \mathrm{~atm}, T(0)=300 \mathrm{~K}, \phi=0.7)$. 


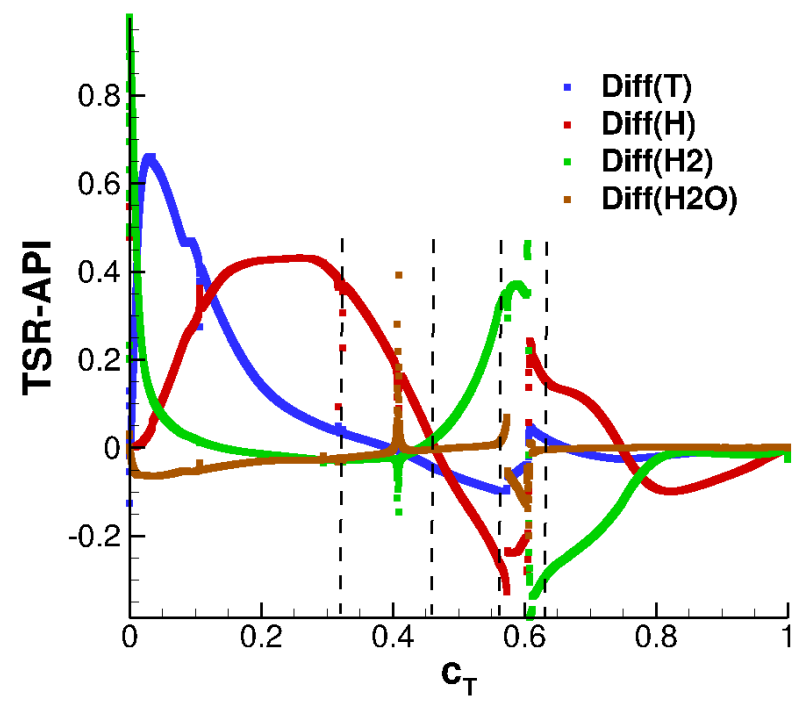

(a)

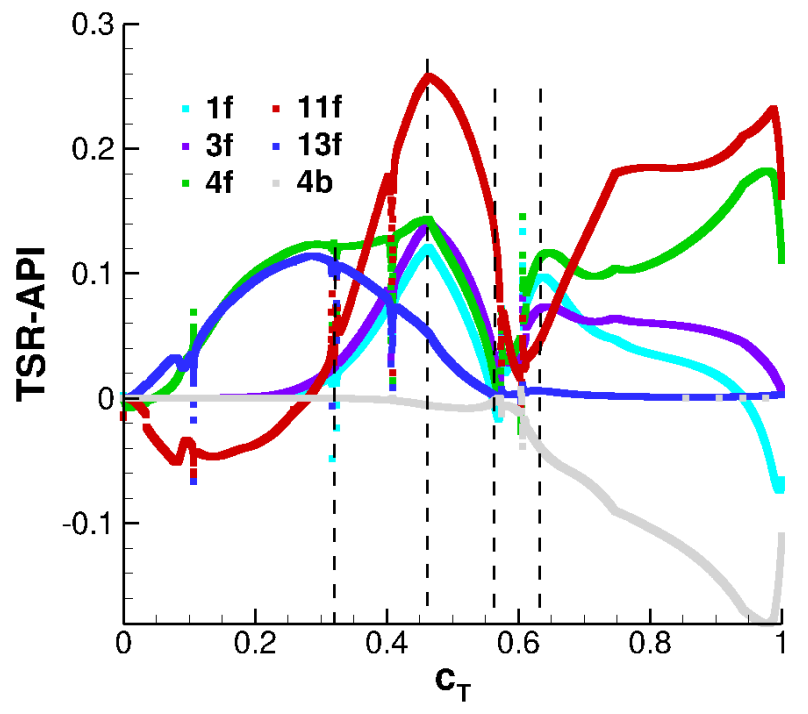

(b)

Figure 7: Spatial profiles of the largest TSR-API contributions from diffusive (left) and kinetic (right) processes, for the laminar flame $(p(0)=1 \mathrm{~atm}, T(0)=300 \mathrm{~K}, \phi=0.7)$.

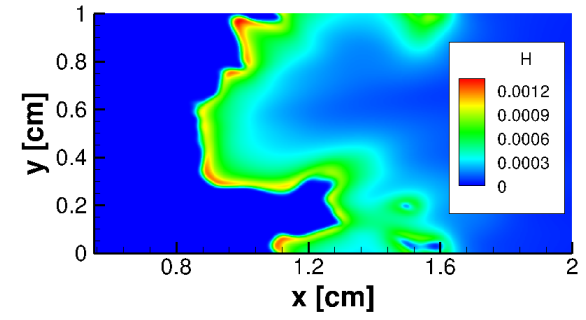

(a)

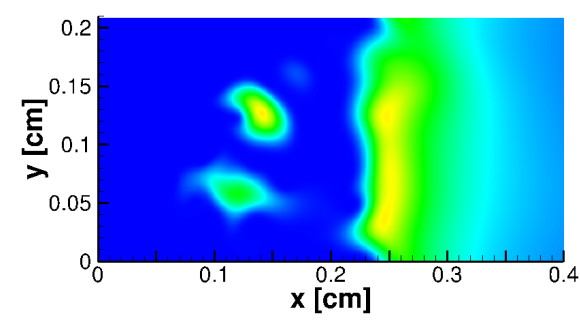

(d)

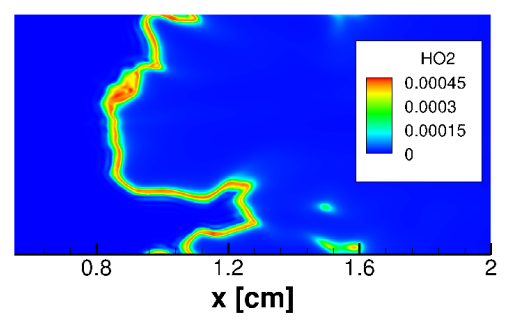

(b)

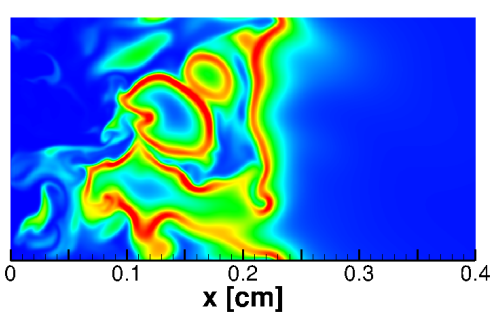

(e)

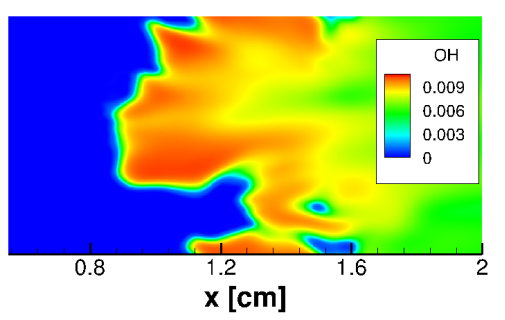

(c)

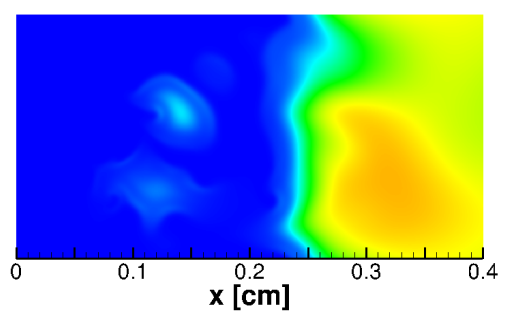

(f)

Figure 8: The spatial distribution of mass fraction of $(\mathrm{a}, \mathrm{d}) \mathrm{H},(\mathrm{b}, \mathrm{e}) \mathrm{HO}_{2}$ and $(\mathrm{c}, \mathrm{f}) \mathrm{OH}$ for Case 2 (upper row) and Case 4 (lower row). 


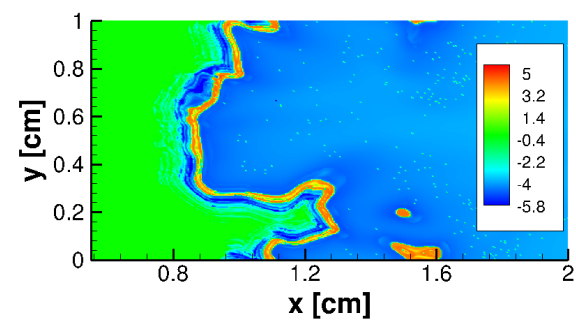

(a)

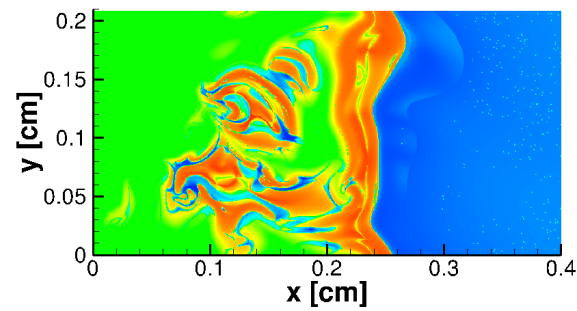

(d)

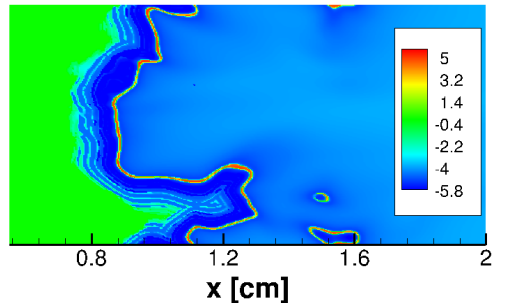

(b)

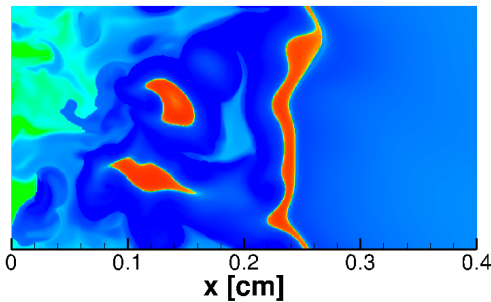

(e)

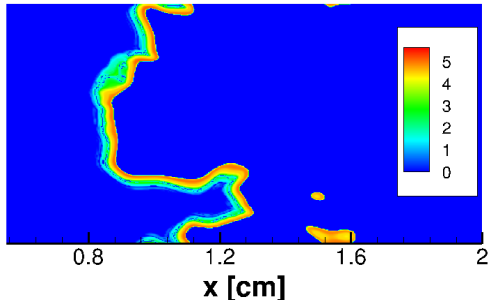

(c)

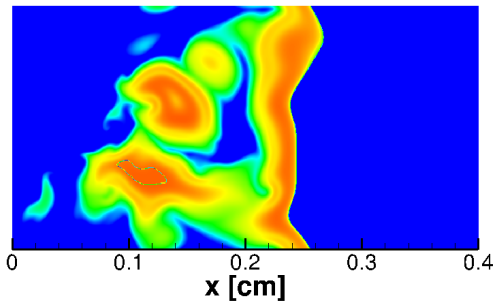

(f)

Figure 9: The spatial distribution of (a,d) $\Omega_{r+d}$, (b,e) $\Omega_{r}$ and (c,f) $\Lambda_{e, f}$ for Cases 2 (upper row) and 4 (lower row).

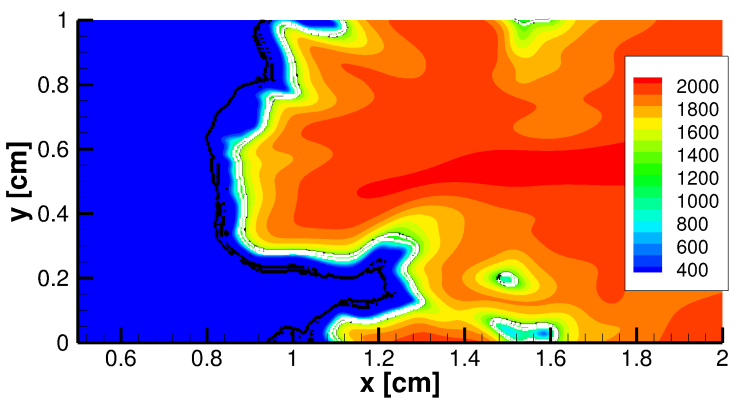

(a)

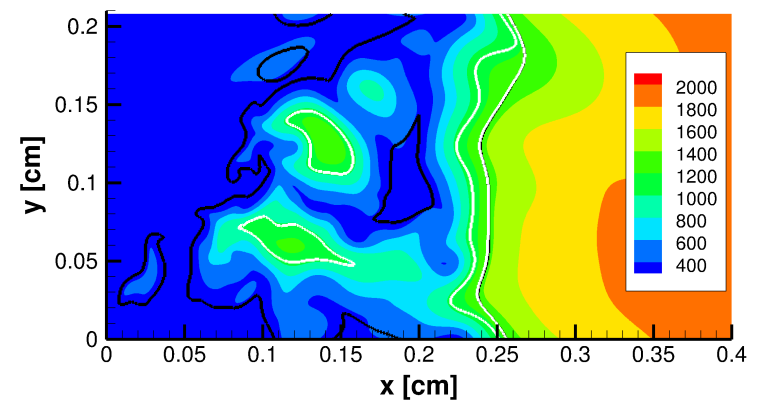

(c)

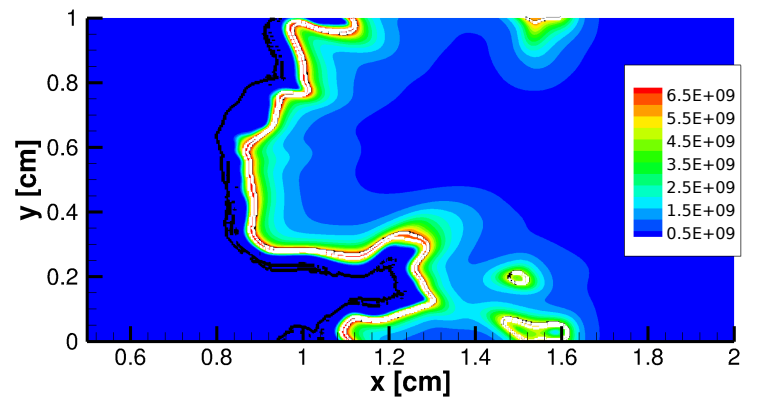

(b)

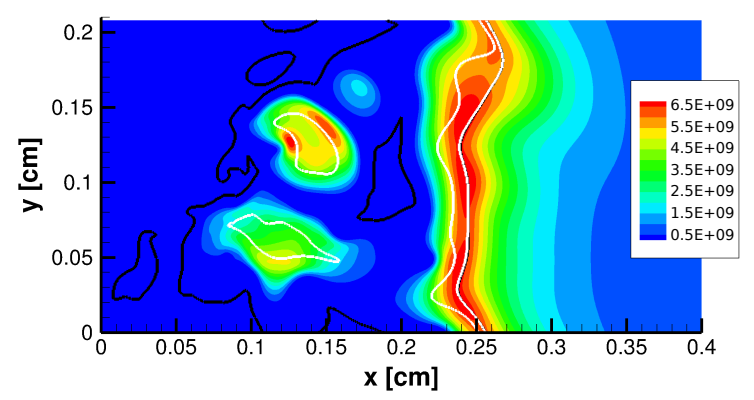

(d)

Figure 10: The spatial distribution of $(\mathrm{a}, \mathrm{c})$ temperature $(\mathrm{K})$ and $(\mathrm{b}, \mathrm{d})$ heat release rate overlaid with the $\Omega_{r}=0$ (white solid lines) and $\Lambda_{e, f}=0$ contour lines (black solid lines), for Case 2 (upper row) and Case 4 (lower row). 


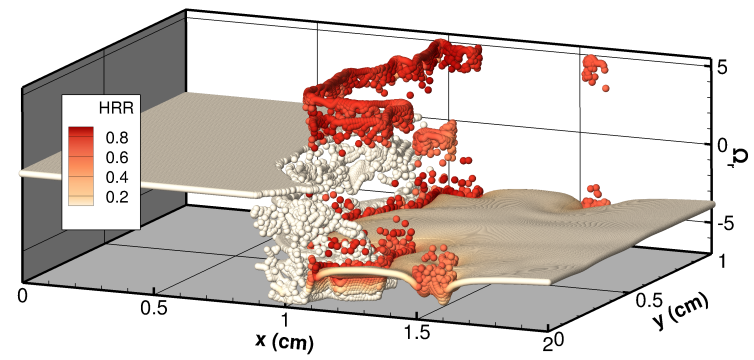

(a)

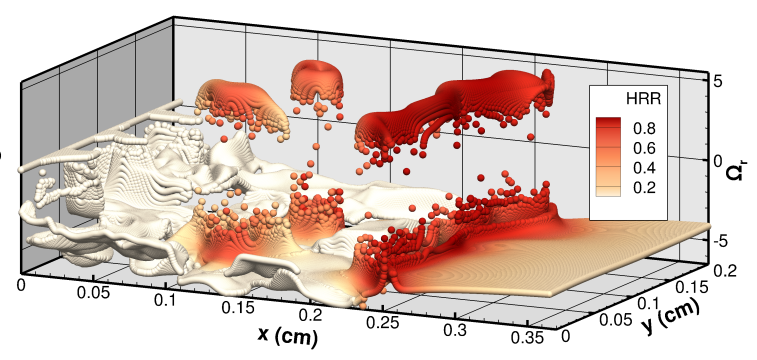

(b)

Figure 11: The height of each scatter plot is proportional to the magnitude of $\Omega_{r}$, while the color legend is associated with the value of HRR; Left (a) is for Case 2; Right (b) is for Case 4. In both cases, the values of HRR have been normalized by their maximum values.

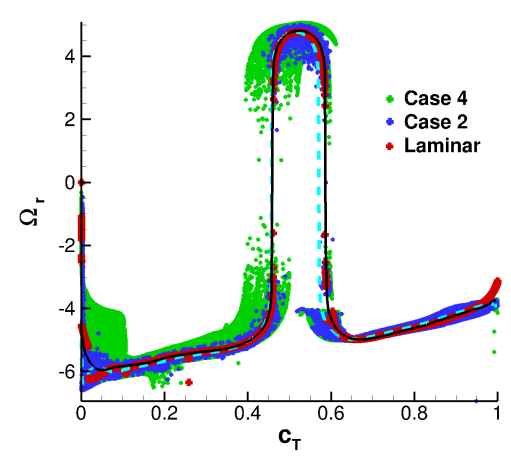

(a)

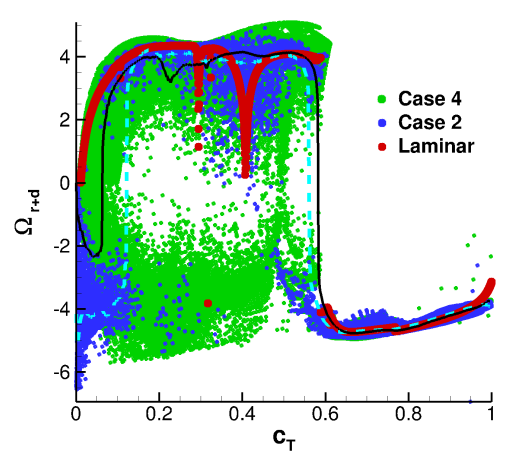

(b)

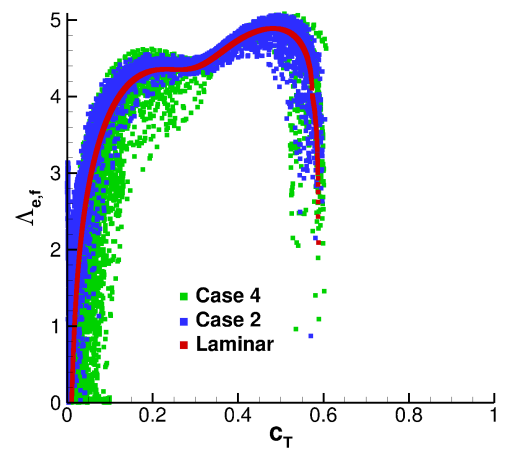

(c)

Figure 12: Scatter plots of $\Omega_{r}$ (a), $\Omega_{r+d}$ (b) and $\Lambda_{e, f}$ (c) against $c_{T}$, for laminar (red), thin reaction zone regime (Case 2 in blue) and distributed combustion regime (Case 4 in green). The conditional averages of $\Omega_{r}$ (left) and $\Omega_{r+d}$ for Case 2 and 4 are overlaid with cyan-dashed and black-solid lines, respectively.

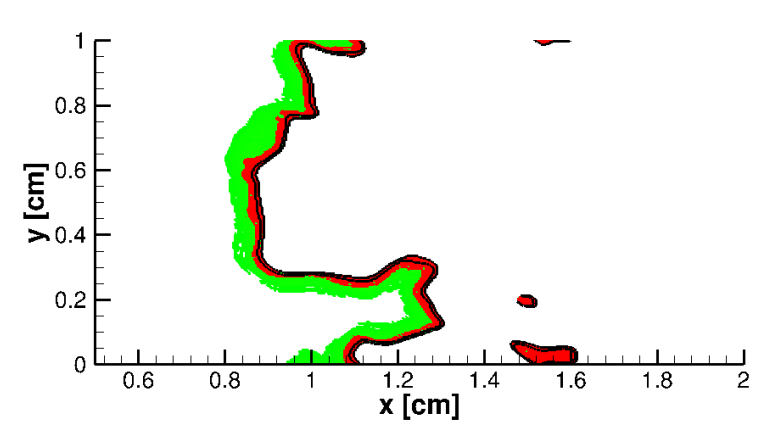

(a)

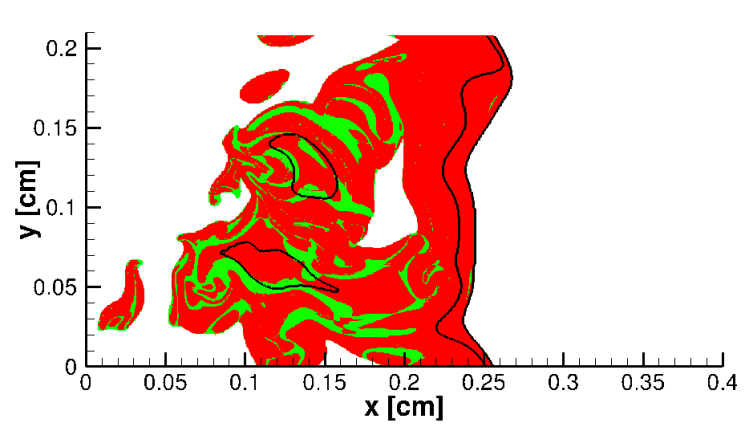

(b)

Figure 13: The contour lines of $\Omega_{r+d}>0$ (in red) and $\Lambda_{e, f}$ (green) overlaid with the $\Omega_{r}=0$ isoline (black), for Case 2 (a) and Case 4 (b). 


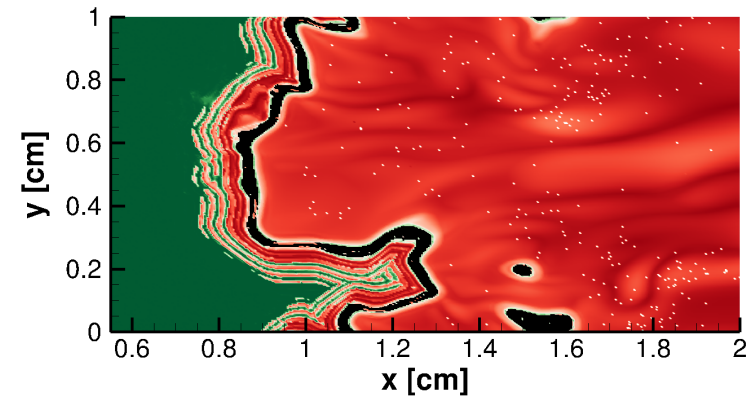

(a)

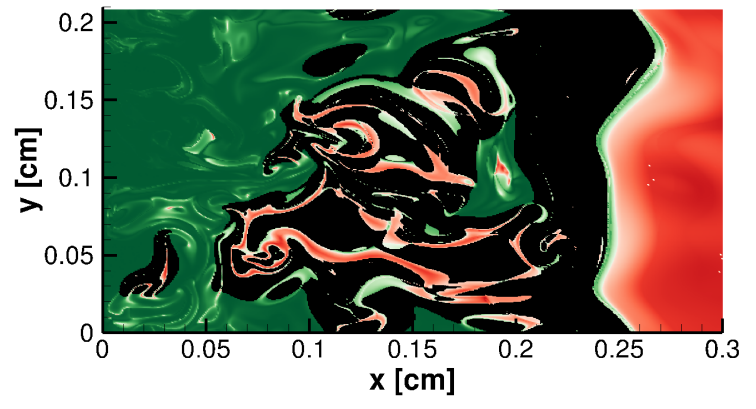

(b)

Figure 14: The distribution of $\Sigma_{r}>50 \%$ (red) and $\Sigma_{d}>50 \%$ (green) overlaid with the contour lines of $\Omega_{r+d}>0$ (in black), for Case 2 (a) and Case 4 (b). The darkness of each color is representative of the intensity of the relevant quantity. 